ISSN: 0212-0267

DOI: http://dx.doi.org/IO.I420I/hedu2OI534IO3138

\title{
EL DISCURSO PEDAGÓGICO EN LA FIESTA DE LA CIENCIA EN LA UNIVERSIDAD DESEADA: ESPAÑA 1900-1936
}

\section{Pedagogic discourse in the science festival in the desired university: Spain 1900-1936}

José María Hernández Díaz

Universidad de Salamanca

Correo-e: jmhd@usal.es

Recepción: I8 de mayo de 20I5. Envío a informantes: 20 de mayo de 2015.

Fecha de aceptación definitiva: 7 de julio de 2015

Resumen: En los inicios del siglo xx la Universidad española ofrece un panorama esperanzador sobre su proceso de modernización científica y docente, tal como lo habían intuido Giner de los Ríos y la Institución Libre de Enseñanza. Era la universidad deseada, como contrapunto a un modelo de universidad anacrónica que se había heredado del siglo xIx. El artículo busca explicar este proceso mediante el análisis de los discursos pronunciados en la llamada Fiesta de la Ciencia.

Palabras Clave: universidad deseada; España; siglo xx; modernización; discurso pedagógico; fiesta de la ciencia.

AвSTRACT: In the early twentieth century the Spanish University offers an encouraging overview of the process of scientific and educational modernization, as they had intuited Giner de los Ríos and the Institución Libre de Enseñanza. It was the Desired University, as a counterpoint to an anachronistic model university which had inherited the nineteenth century. The article seeks to explain this process by analyzing speeches delivered in the so-called Science Festival.

KeY WORDs: desired university; Spain; 20 $0^{\text {th }}$ century; modernization; Pedagogic discourse; science festival. 
EL DISCURSO PEDAGÓGICO EN LA FIESTA DE LA CIENCIA

EN LA UNIVERSIDAD DESEADA: ESPAÑA I9OO-I936

JOSÉ MARÍA HERNÁNDEZ DÍAZ

\section{Introducción}

L

A «UNIVERSIDAD DESEADA» es la expresión de un concepto modernizador de universidad y de educación superior que defienden varios universitarios e intelectuales españoles de principios del siglo xx, una universidad distinta que se convierte para muchos de ellos en un anhelo ${ }^{\mathrm{I}}$. Unamuno, Giner de los Ríos y Cajal pueden representar perfectamente este movimiento universitario reformador, cada uno a su estilo, como grandes hitos y referentes, en forma de trilogía casi mítica.

Pero también puede aplicarse esa aspiración a otro tipo de universidad, y ese deseo de universidades modernas y dinámicas, a decenas de profesores e investigadores universitarios que en la España del primer tercio del siglo xx aspiran a contribuir a la construcción de una universidad distinta, abierta a Europa y al mundo, capaz de formar buenos profesionales, menos retórica y formalista, más libre y democrática, más asentada en la producción de conocimiento, en la investigación, y con capacidad de ofrecer respuestas convincentes a los problemas de la industria, de la sociedad y de las comunidades a las que sirven y donde se insertan.

Ese afán reformador y regeneracionista de la educación superior, de la investigación, y también en buena medida del sistema educativo, cuaja de forma principal y eficaz por el impulso y en la articulación de un organismo que había sido soñado por alguno de estos insignes intelectuales, principalmente por Giner, y que fue presidido durante años por Santiago Ramón y Cajal. Nos referimos, claro está, a la Junta para Ampliación de Estudios, organismo público impulsor de lo más granado y original de la ciencia española, de la cultura, y en parte de diferentes mejoras educativas de la España que va desde i9oo hasta el inicio de la confrontación bélica de $1936^{2}$.

La expresión «la universidad deseada» la hemos utilizado por primera vez hace ya algún tiempo para referirnos a la Universidad de Salamanca de estos mismos años ahora estudiados, empeñada en impulsar cambios notorios respecto a la herencia recibida del imperio del reglamento universitario del siglo xix, $c f r$. Hernández Díaz, José María: «Entre las resistencias al cambio y la universidad deseada, I900-I936», en Rodríguez-San Pedro BeZares, Luis Enrique: Historia de la Universidad de Salamanca. Vol. I. Trayectoria y vinculaciones, Salamanca, Ediciones Universidad de Salamanca, 2002, pp. 283-312.

Cfr. Sánchez Ron, José Manuel (coord.): La Junta para Ampliación de Estudios e Investigaciones Científicas 80 años después, Madrid, csic, 1988, 2 vols.; Peset Reig, José Luis y Hernández SANDoicA, Elena: «Instituciones científicas y educativas», en Historia de España. Ramón Menéndez Pidal. Tomo XXIX 2. La Edad de Plata de la cultura española (1989-1936), Madrid, Espasa Calpe, 1996, pp. 545-580; Peset Reig, Mariano: «Centralismo y autonomía en las universidades (siglos xix y xx)», en SEDHE: La Universidad en el siglo XX (España e Iberoamérica), Murcia, Universidad de Murcia, i998, pp. 25-34; Gamero Merino, Carmela: «Castillejo a través de su correspondencia como secretario de la JAE», Historia de la Educación, 5 (1986), pp. 375-40o; Hernández DíAz, José María: «La Junta para Ampliación de Estudios y las universidades españolas», en Hernández Díaz, José María: De la Junta para la Ampliación de Estudios a la construcción del Espacio Europeo de Educación Superior, Salamanca, Globalia Ediciones Anthema, 2009, pp. 17-40; Hernández Díaz, José María: «El modelo de la universidad alemana en España», en Hernández DíAz, José María (coord.): La pedagogía alemana en España e Iberoamérica (I8I0-20I0), Valladolid, Castilla Ediciones, 20II, pp. 225-247. La bibliografía sobre el tema, por fortuna, es hoy abundante. 
EL DISCURSO PEDAGÓGICO EN LA FIESTA DE LA CIENCIA

EN LA UNIVERSIDAD DESEADA: ESPAÑA I9OO-I936

JOSÉ MARÍA HERNÁNDEZ DÍAZ

La lucha en pro de una universidad novedosa y diferente a la napoleónica, burocrática e inmovilista recibida del siglo anterior ${ }^{3}$, y sobre todo científicamente capaz y abierta a las demandas de la sociedad española, va erigirse en el hilo conductor de muchas iniciativas de reforma que se emprenden, y explica el deseo profundo de cambio hacia otro tipo de educación superior. Por ello el movimiento de crítica a la universidad anterior es potente y continuado, aunque discontinuo por momentos, y las propuestas alternativas van emergiendo de forma paulatina en diferentes universidades.

Una viva expresión de lo que indicamos sobre esa universidad deseada es la posición adoptada por un selecto grupo de catedráticos, de diferentes facultades y especialidades españolas, que permanece bien reflejada en sus expresiones públicas, en sus discursos, y en concreto en el discurso académico de la llamada «fiesta de la ciencia» que se celebra con carácter anual en todas las universidades españolas de estas décadas primeras del siglo xx. La retórica en esta ocasión nos permite incursionar en la comprensión interna de pareceres y debates que se viven con intensidad en los claustros universitarios y en los paraninfos de una España tan requerida de cambios profundos y de regeneración real.

Hoy es obvio que para adentrarnos en la historia de la universidad española del primer tercio del siglo xx (1900-1936) podemos servirnos de diferentes fuentes e instrumentos de apoyo propios de la historiografía de nuestro tiempo, tales como la iconografía, la información hemerográfica de la prensa diaria y de las revistas científicas, los enormes fondos documentales conservados en diferentes archivos universitarios y bibliotecas de las universidades más antiguas en esas fechas, los discursos parlamentarios, la información legislativa específica propia de los boletines de instrucción pública, las estadísticas y tantos otros referentes ubicados en archivos y fundaciones particulares, archivos y bibliotecas municipales, provinciales, de Diputaciones. Podríamos añadir aún otras vías propias de la documentación oral, audiovisual, de las fuentes materiales, espaciales y de su arquitectura. Son todas ellas opciones complementarias para una elección explicativa que solo pueden ser útiles para enriquecer y completar la historia de la Universidad española de ese momento tan particular, tan esperanzador y creativo, que se interrumpe de forma violenta y desastrosa con el inicio de la guerra civil en julio de 1936 .

Dentro de las fuentes escritas, de procedencia universitaria, como luego vamos a exponer in extenso, que nos facilitan una aproximación hermenéutica al tipo de universidad que se desea construir y conquistar en un afán decidido de modernización y reforma universitaria, encontramos el discurso académico de inauguración de curso que se pronuncia de forma secuenciada el primer día de cada año académico por parte de un catedrático de una de las facultades de la universidad de referencia, entre cada una de las existentes. No hablamos ahora de los discursos de política universitaria que pronuncian rectores, ministros o políticos invitados al acto, que seguramente merecen otro tratamiento por parte

Cfr. Hernández Díaz, José María: «Francia en la universidad de la España contemporánea», en Hernández Díaz, José María (ed.): Francia en la educación de la España contemporánea (i8082008), Salamanca, Ediciones Universidad de Salamanca, 20II, pp. 323-344. 
del historiador de las universidades, sino del discurso elaborado y pronunciado por un académico en un marco no menos honorable y lleno de dignidad, y a veces boato, como es el paraninfo el día de la inauguración del curso académico de una universidad.

Ese formato de intervención universitaria, propio del mundo de los honores y distinciones universitarias, revestido de ceremonial y liturgia, que tanto resuenan de las pautas eclesiásticas precedentes y muy arraigadas, tradicionales en Europa, es adoptado en España, desde poco después de la reforma del Plan Pidal de I845, por todas las universidades entonces existentes y oficiales (estamos hablando de las de Salamanca, Valladolid, Santiago, Sevilla, Barcelona, Valencia, Granada, Sevilla, Zaragoza, y la de Madrid o Universidad Central, que había nacido al desaparecer la histórica de Alcalá en I836, todas ellas procedentes del siglo XIX, y las creadas en Murcia-1912- y La Laguna-1926-).

\section{El discurso académico en la historia de la llamada fiesta de la ciencia}

En el mundo académico latino, y en España en concreto, hemos recibido una larga y rica tradición retórica, que se configura con esplendor y excelencia en el mundo grecolatino, y que se ha convertido en uno de los muchos y valiosos legados culturales y científicos que Grecia primero y Roma después dejan para Occidente. Asociada a éxito en la vida democrática y en la controversia jurídica, y a exposición pública de saberes y puntos de vista, la retórica se fue erigiendo en un instrumento imprescindible de la vida cultural y científica de las polis griegas, desde Gorgias y los sofistas hasta el gran maestro del discurso en el mundo griego clásico, Isócrates. Combatida por la filosofía, la retórica no obstante es reconocida como instrumento imprescindible del éxito en la controversia pública, fuera ésta política, jurídica o intelectual.

Roma y sus mejores exponentes de la oratoria (Cicerón y Quintiliano, por ejemplo) ratificarán las prácticas retóricas de los antecesores griegos y buscan una mejor aplicación pedagógica y jurídica. Los romanos eran receptores directos de la tradición helenística que incluía la retórica en el mínimo cultural de la persona poseedora de saber suficiente para triunfar en los negocios y en la vida política, para alcanzar prestigio y reconocimiento social. El helenismo llamaba a esta síntesis de los saberes la «enkiclios paideia», conocimiento enciclopédico, y dentro del mismo resultaba imprescindible el estudio y el dominio de la retórica.

Más tarde, durante el largo milenio medieval, incluso en la etapa renacentista, el dominio de la retórica fue patrimonio casi exclusivo de los eclesiásticos, en monasterios, órdenes religiosas y clero secular, en el ámbito de la oratoria litúrgica, en la predicación y en algunas actuaciones jurídicas aisladas. El discurso queda recluido a los espacios, en las manos y poder del clero culto, bien para redactar argumentos luego leídos y proclamados por otros sacerdotes, bien para ser utilizado y ornamentado por los grandes oradores en ocasiones especiales. Pero también la formación retórica era parte imprescindible de la formación de todo universitario en sus estudios menores, en la Facultad de Artes, como un saber y conocimiento previo a los estudios superiores. 
EL DISCURSO PEDAGÓGICO EN LA FIESTA DE LA CIENCIA

EN LA UNIVERSIDAD DESEADA: ESPAÑA I9OO-I936

JOSÉ MARÍA HERNÁNDEZ DÍAZ

Todavía en la etapa de la Ilustración se va a mantener en Europa este tipo de prácticas litúrgicas y oratorias del clero y de algunos universitarios, si bien comienzan a redactarse otro tipo de discursos y proclamas que nos remiten más a pensamientos ordenados, ensayos, reflexiones para uso público. De tal manera que ese modelo de discurso próximo a la liturgia eclesiástica se traslada poco a poco al ceremonial universitario, donde conviven espacios procesionales, oratorios, vestimentas, ceremoniales, loas, honras. En el siglo xviII encontramos ejemplares expresiones de este tipo de prácticas ceremoniales y discursos dentro de universidades, como la de Salamanca, por ejemplo.

De tal forma que cuando se produce la reforma liberal de la universidad en España, representada en el Decreto del marqués de Pidal de I845, en las décadas anteriores del siglo xix se mantiene aún muy viva la práctica del discurso inaugural del año académico dentro del ceremonial que da inicio a las actividades lectivas de las universidades.

Lo que ocurre desde la segunda mitad del siglo xIx, y se mantiene hasta nuestros días en las universidades españolas, es que en el ceremonial de inicio de cada año lectivo (la llamada por algunos «fiesta de la ciencia») se contempla año tras año la presencia de un discurso inaugural, pronunciado por un catedrático de prestigio reconocido, y en activo dentro de la universidad. El acuerdo más o menos implícito en la forma de concebirlo es que fuera rotatoria la presencia de las diferentes facultades y estudios universitarios y, ante todo, que el discurso fuera de alta calidad y de orientación pedagógica, próximo a los intereses de los estudiantes y asistentes, capaz de proponer nuevas formas de enseñanza en un capítulo concreto de la ciencia, en una disciplina o su ámbito de influencia.

Éste es el asunto que nos llama a nosotros a la reflexión en esta ocasión, la pregunta por el contenido y orientación pedagógica de los discursos académicos pronunciados en la Universidad española entre 1900 y 1936 el día de la inauguración del curso y a cargo de un catedrático perteneciente al claustro. El académico que imparte la lección magistral (clara resonancia a la lectio medieval) ha de ejercer, ha de estar activo en alguna de las Facultades de Derecho, Medicina, Ciencias, Letras y Farmacia de su Universidad, las únicas por entonces reconocidas como universitarias, pues las Escuelas Técnicas en esa época (y hasta la reforma de 1970) quedaban fuera de la Universidad en el modelo español.

Con anterioridad a nosotros, algunos estudios, como el de Rubio y Borrás han prestado su atención al tema del discurso universitario en la Universidad de

4 Nos parece de interés conocer el punto de vista de este historiador y bibliotecario de Barcelona, «Entre las festividades académicas que se celebran en las universidades españolas, ninguna refleja de modo más fiel la vida escolar como las que se conocen con el nombre de "Aperturas de curso". Son el comienzo de una nueva era: el cambio de orientación respecto a los cursos anteriores: la perspectiva de un vasto campo en el que la inteligencia ha de sembrar semilla de indiscutibles y provechosos frutos que han de influir poderosamente en el derrotero de la sociedad humana.

Para el maestro cuya cátedra no es al automatismo que sirve de pretexto al cobro de la asignación que el Estado le asigna, repitiendo todos los años en los mismos días y casi en las mismas horas, las mismas palabras, como el ignorante predicador del inmortal P. Isla, un nuevo curso es la manifestación de aquellas nuevas ideas con que ha enriquecido su arsenal científico y literario; para el alumno aprovechado al que la universidad no es un mero pasatiempo o un lugar de hastío, el curso que empieza, es el peldaño más que sube, en aquella escala que ha de conducirle a la obtención del título que le servirá de arma de combate en la difícil lucha por la existencia. 
Barcelona del siglo xix, en el seno de otros trabajos relativos a acreditar la restauración de aquella universidad catalana por parte de la política de los liberales, después de la dolorosa experiencia del traslado a Cervera de la primera universidad creada en Barcelona (I450).

Gómez Izquierdos, catedrático de Granada, dedica precisamente su lección de apertura de curso de 1920 a lo que él llama más expansivamente «fiesta de la labor intelectual», o lo que hemos denominado nosotros «fiesta de la ciencia» en expresión de lo admitido en el siglo xIx, en la cual el discurso del catedrático elegido o nombrado para la ocasión desempeña una posición central en los actos ceremoniales.

También encontramos tratamientos sobre el tema en la Universidad de Salamanca del XIx, si bien de forma indirecta, caso de Lamberto de Echeverría ${ }^{6}$, o de manera explícita y directa como nosotros mismos hemos escrito para el tercio central de esa centuria ${ }^{7}$; para otros años diferentes en otras universidades, como sucede en la de Zaragoza con el trabajo de Rosa Domínguez Cabrejas. ${ }^{8}$ No es menos cierto que en diferentes estudios particulares de universidades españolas sobre el siglo XX se han utilizado los discursos de inauguración del año lectivo como fuente y documento de información, en diferentes contextos, caso de Rosa Calatayud para Valencia ${ }^{9}$ hace algunos años, y también nosotros para fines del XIX y este primer tercio del siglo xx en Salamanca, y sobre el asunto concreto de la libertad de enseñanza desde una lectura universitaria ${ }^{10}$. Más recientemente desde la Universidad de Oviedo se ha trabajado el tema de los discursos inaugurales pudiendo ofrecer una más que meritoria recopilación de los de su universidad entre 1862 y 1903 $3^{\mathrm{II}}$.

Por eso, en aquellas festividades, se congregan en los respectivos paraninfos, verdaderos templos de Minerva, los catedráticos y alumnos que sienten en su corazón la vida universitaria, estableciéndose moralmente el pacto de cariño que ha de ser el lazo de unión durante los meses que dura la enseñanza oficial.

Extraordinaria importancia han revestido las inauguraciones de estudios en las universidades españolas en todas las épocas; y triste es confesarlo, únicamente en los tiempos modernísimos, cuando el mercantilismo y las miras egoístas dominan en todo el orden social, penetrando hasta en el inviolable del saber, aquella solemnidad con que se celebraban va decayendo, reduciéndose casi meramente a un acto oficial al que suelen asistir reducido número de doctores», en RUBIO Y BorRÁs, Manuel: Aperturas de curso en la Universidad de Barcelona desde 1536 a I9Ig. Reseña histórica y bibliográfica, Barcelona, Editorial Cultura, 1919, p. 15.

Cfr. Gómez IzQuierdo, Alberto: La inauguración solemne del curso como fiesta de la labor intelectual. Discurso pronunciado en la Universidad de Granada en la inauguración del curso 1920 a I92I, Granada, Tip. López Guevara, 1920, p. 47.

6 Cfr. EcheverRía, Lamberto de: De oratoria universitaria salmantina. Oración pronunciada en la solemne apertura del cuso 1977-1978 en la Universidad de Salamanca, Salamanca, Ediciones Universidad de Salamanca, 1977.

Cfr. Hernández Díaz, José María: «La pedagogía en la fiesta de la ciencia de la Universidad de Salamanca (1845-1874)", REDEX. Revista de Educación de Extremadura, 4 (2012), pp. 49-69.

8 Cfr. Domínguez Cabrejas, Rosa: «El discurso pedagógico a través de los paraninfos. Universidad de Zaragoza (I900-1923)», en x Coloquio de Historia de la Educación: La Universidad en el siglo XX. España e Iberoamérica, Murcia, SEDHE/Universidad de Murcia, I998, pp. I23-I3I.

9 El trabajo se presentó en el I Coloquio de Historia de la Educación en España, celebrado en Alcalá en 1982 , pero no nos consta que se haya publicado.

1o Cfr. Hernández Díaz, José María: «La libertad de enseñanza en la Restauración y su incidencia en la Universidad de Salamanca», Historia de la Educación, Salamanca, 3 (1984), pp. Io9-I26.

"Cfr. El Grupo de Oviedo: Discursos de apertura de curso de la Universidad de Oviedo (I862I903), Estudio preliminar y edición, Oviedo, Universidad de Oviedo, 2002, 2 vols. 
EL DISCURSO PEDAGÓGICO EN LA FIESTA DE LA CIENCIA

EN LA UNIVERSIDAD DESEADA: ESPAÑA I9OO-I936

JOSÉ MARÍA HERNÁNDEZ DÍAZ

El modelo retórico utilizado no es originario de esta etapa histórica que ahora nosotros estudiamos, pues al menos desde el ceremonial de i719 se contempla y practica cada I8 de octubre una lección «de principio», acompañada entonces de la profesión de fe tridentina de los catedráticos, seguida de misa. Parece que son pautas ceremoniales procedentes en su origen de las universidades del Reino de Aragón y más tarde extendidas al resto.

Por otra parte, algo que define bien el escaso grado de dedicación a la investigación por parte de los profesores de la Universidad española todavía en el primer tercio del siglo xx, es que con frecuencia este discurso es la «opera omnia» de un catedrático para toda su vida, es decir, que no escribe más textos ni monografías en toda su vida. Otros ni siquiera leen discurso, viven y mueren ágrafos. Bien es verdad que, justamente en esta etapa de la historia universitaria española, comienzan a aparecer signos nuevos, pautas de investigación que expresan la búsqueda de una nueva universidad, que desean otra universidad, seguramente muy influenciados por la experiencia transformadora y modernizadora procedente de Europa, y en parte movilizada por la Junta para Ampliación de Estudios a partir de 1907 .

Hay que aclarar que el modelo de retórica universitaria es muy parecido en todas las universidades españolas del siglo xix, y en todas se pronuncia cada año un discurso de esta clase en los inicios del curso académico. Había que seguir y mantener las directrices y orientaciones oficiales de los gobiernos liberales. El altisonante y retórico texto del catedrático salmantino de griego Cándido Herrero Garrido $^{\mathrm{I} 2}$, pronunciado en 1859 en un lenguaje propio de una arenga casi militar, nos ponía en situación sobre el sentido de estos discursos inaugurales de las universidades españolas.

\section{La universidad en la España del primer tercio del siglo $\mathrm{xx}$}

Si hay algo que identifique con claridad a la universidad española del primer tercio del siglo xx es su tensión permanente entre la pervivencia de anacronismos heredados del modelo universitario vigente en el siglo xIx y el ansia de reforma y modernización, buscando adecuarse al cambio de modelo de universidad y de institución de educación superior que comienza a operarse en todo el mundo, al menos en el más avanzado.

Sheldon Rothblatt y Böjrn Wittrock ${ }^{13}$ hace algunos años han estudiado de forma acertada para países y espacios culturales de la geopolítica anglosajona y nórdica ese proceso de cambio, o cómo han comenzado a integrarse dos grandes tradiciones y modelos de universidad. Por una parte, la que forma profesionales al servicio de las administraciones públicas y atentos a las demandas reales del mercado y la sociedad, y puede adoptar dos pautas distintas, como es la tradicional

${ }_{12}$ Cfr. Herrero Garrido, Cándido: La historia de la educación de la inteligencia. Discurso inaugural en la Universidad de Salamanca pronunciado el I de octubre de I859 para el curso I859 a I860, Salamanca, Imprenta de Diego Vázquez, I859.

${ }_{13}$ Cfr. Rothblatt, Sheldon y Witrock, Björn: La universidad europea y americana desde I80o. Las tres transformaciones de la universidad, Barcelona, Pomares, 1996. 
francesa y la inglesa. Por otra, el concepto de universidad humboldtiana que nace y se consolida en Berlín a partir de i8ıo y se significa como una institución poderosa para el cultivo de la ciencia, para el fomento de la investigación, para la formación de futuros investigadores. Las universidades van a ir ocupando un espacio día a día ampliado para la producción de ciencia básica, y en muchos casos tecnología.

Así mismo, en los países del sur de Europa se han producido avances y estudios para sus universidades en el cambio de siglo y hasta la llegada de la II Guerra Mundial, como los que han merecido Francia por parte de Jacques Verger y Christophe Charle ${ }^{14}$, de Luis Reis Torgal para las universidades de Portugalis, Antonio Santoni Rugiu para la universidad en Italia de los dos últimos siglos ${ }^{16}$ y diferentes trabajos para las universidades de España ${ }^{17}$.

En el caso español se constata todavía una fuerte persistencia del modelo napoleónico de universidad, y por ello también una mayor resistencia al cambio, y un deseo casi estructural de afincarse en el inmovilismo. No obstante, el movimiento del regeneracionismo, los krausistas e institucionistas con Giner de los Ríos $^{18}$ al frente, y personalidades universitarias brillantes como puedan ser las señaladas en Unamuno ${ }^{19}$ y Ramón y Cajal ${ }^{20}$ hacen caminar, aunque con lentitud,

${ }^{14}$ Cfr. Verger, Jacques: Histoire des universités en France, Toulouse, Éditions Privats, I986; Charle, Christophe y Verger, Jacques: Histoire des universités, XIIé-XXIe siècle, Paris, PUF, $20 I 2$.

${ }_{15}$ Además de otros trabajos del autor principal, remitimos a la consulta de $c f r$. TorgAL, Luis Reis y Brigato Esther, Angelo: Que universidade? Interrogações sobre os caminos da universidade em Portugal e no Brasil, Coimbra, Imprensa Universitaria da Universidade de Coimbra, 2014.

i6 Cfr. Santoni Rugiu, Antonio: «Líneas de desarrollo y contradicción en la universidad italiana de los dos últimos siglos», Historia de la Educación, Salamanca, 5 (1986), pp. II9-I30.

${ }_{7}$ Todas las universidades españolas, públicas y procedentes del siglo xix, han merecido los correspondientes estudios históricos. Es decir, cada universidad tiene su historia, y dentro de ella se ha estudiado la etapa que ahora nos ocupa, y a la que remitimos, por lo que aquí no podemos entrar en detalles de cada una de ellas.

Alguna referencia de carácter general, además de las mencionadas para la JAE, puede servir, $c f r$. Peset, Mariano: «Política universitaria tras el desastre del 98», en Rodríguez-San Pedro, Luis Enrique (ed.): Las universidades hispánicas: de la monarquía de los Austrias al centralismo liberal. Vol. II, Salamanca, Junta de Castilla y León, 2000, pp. 425-447; Peset Reig, Mariano y Baldó, Marc: «Pasado y futuro de las universidades de la Corona de Aragón», en Busqueta, J. J. y Pemán, J. (coords.): Les universitats de la Corona de Aragón, abir i avui. Estudis historics, Barcelona, Pòrtic, 2002, pp. 563-602; HeRnández SANDOICA, Elena: «Los archivos universitarios y la historia de las universidades: perspectiva de los investigadores», en Archivos universitarios e historia de las universidades, Getafe, Universidad Carlos III, 2003, pp. 97-I29; Hernández DíAz, José María: «La universidad en España del Antiguo Régimen a la LRU (1983)», Aula. Revista de Pedagogía de la Universidad de Salamanca, 9 (1997), pp. I9-44; IDEM: «L'université dans l'Espagne contemporaine (I8I2-I983)», Histoire de l'Education, Paris, 78 (I998), pp. 3I-56.

Para consulta bibliográfica in extenso y del estado de la cuestión de este tema, remitimos a los capítulos correspondientes de Guereña, Jean Louis; Ruiz Berrio, Julio y Tiana Ferrer, Alejandro (eds.): Nuevas miradas historiográficas sobre la educación en la España de los siglos XIX y XX, Madrid, Ministerio de Educación, 20Io.

i8 Cfr. Giner de los Ríos, Francisco: Escritos sobre la universidad, edición de Teresa Rodríguez de Lecea, Madrid, Espasa Calpe, 1990. Los escritos fueron originales del autor en el último cuarto del XIx y algunos a inicios del xx.

is La bibliografía sobre Unamuno en relación a este tema es muy abundante y actualizada. Sin entrar en detalles, tal vez sus posiciones en torno a la Asamblea Universitaria de Barcelona de 1905 puedan ser lo más representativo.

${ }_{20}$ Cfr. Ramón y Cajal, Santiago: Recuerdos de mi vida, Madrid, Impr. Juan Pueyo, 1923. 
EL DISCURSO PEDAGÓGICO EN LA FIESTA DE LA CIENCIA

EN LA UNIVERSIDAD DESEADA: ESPAÑA I9OO-I936

JOSÉ MARÍA HERNÁNDEZ DÍAZ

hacia una universidad española cada vez más alejada de las posiciones reglamentistas y dogmáticas que caracterizan la Universidad de la España del xIX.

Ahora se pide que la universidad se abra a todos los sectores sociales, que acepte la presencia de las mujeres en las aulas, que los métodos didácticos sean más próximos a los intereses de los estudiantes, que los profesores estudien y se pongan al día en su disciplina científica y no se limiten a exigir conocer de memoria el libro de texto, que los programas de las materias, las orientaciones y contenidos de los saberes estén actualizados, que se establezca otra relación más próxima entre el profesor y el estudiante, que se dote adecuadamente la enseñanza práctica de todas las materias, que se mejoren las instalaciones básicas del estudio y la investigación (como bibliotecas, revistas científicas, laboratorios), que la Universidad esté cada vez más abierta al exterior de España (por lo que resulta fundamental incrementar la dotación del programa de becas y pensionados).

Es muy frecuente escuchar propuestas de una nueva universidad acorde en sus enseñanzas con lo que necesita la sociedad, con el desarrollo económico y con los avances de la ciencia en general.

Se solicita de forma persistente un modelo de gestión y gobierno universitario que retome las tradiciones de autonomía y libertad, de que gozaron en algún momento del pasado las universidades, en el que los implicados de todos los estamentos universitarios puedan gozar de oportunidades democráticas.

Este proyecto de universidad buscada, deseada, anhelada finalmente no fue posible de alcanzar, en lo fundamental, por las muchas alternativas y vaivenes que tuvo la sociedad española en estas décadas, y que caló en una universidad finalmente sensible a los movimientos políticos de la época, como seguramente tenía que producirse. Lo cierto es que el proyecto de cambio universitario más innovador que conocemos de esta etapa, el que en 1933 presentó a las Cortes el ministro de Instrucción Pública y Bellas Artes Fernando de los Ríos, no logró avanzar en el trámite parlamentario, y quedó en el cajón de las aspiraciones fallidas. Aquella universidad deseada no pudo caminar con más rapidez, y quedó relegada para tiempos mejores, porque los peores llegaron en 1936 con el golpe de Estado de los militares que apoyaron a Franco.

\section{La universidad deseada en la retórica pedagógica de la fiesta de la ciencia (1900-1936)}

De todos estos anhelos y proyectos de reforma universitaria conocemos muchos detalles a través de la normativa general, pero conviene adentrarse en aspectos y capítulos más concretos de la vida universitaria. Éste es el caso que nos aporta el estudio de los discursos que se pronuncian en la «fiesta de la ciencia», una vez al año, en cada una de las universidades. El seguimiento y estudio de esta fuente de información, retórica si se quiere, pero aplicada al conjunto de universidades españolas, nos ofrece resultados muy interesantes para comprender por otra vía los avances y sueños que los académicos proponen a sus claustros y a los estudiantes, y de paso a las autoridades políticas que les escuchan. 
Tomando en cuenta el número de universidades existentes en esos años (las del Estado, diez inicialmente, más las de Murcia y La Laguna nacidas después, pues los centros de formación de Deusto y Comillas de la Compañía de Jesús tienen entonces un claro perfil diferenciador, y solo con dificultad pueden ser admitidas como tales universidades), y el número de años que abarca nuestro estudio (I90o a 1935), sabemos que se han pronunciado en las universidades españolas un total de 378 discursos académicos, en la inauguración del curso de las diferentes universidades. Ahora bien, una vez analizados todos ellos, buena parte de los mismos carecen de interés pedagógico o de propuesta de reforma universitaria. La razón no es otra que el mantenimiento de la tradición heredada por parte de muchos profesores, quienes en su discurso se refugian en temas estrictamente técnicos de sus especialidades, y no desean evidenciar ningún compromiso de cambio en su docencia, ni tampoco en el funcionamiento de su universidad.

Nos parece muy revelador y orientativo al efecto lo que indica el catedrático de Medicina de la Universidad de Valladolid Federico Murueta Goyena, al comienzo de su discurso de 1915 , después de disculparse al tener que actuar por reglamento y orden riguroso, para justificar la elección que él hace en su caso.

La primera y más importante dificultad con que tropiezo, arranca de la cuestión ardua de la elección de tema para una oración inaugural, que convenga o sea apropiado a la naturaleza del presente acto académico. Unos, llevados del buen propósito de afirmar aún más sus conocimientos, escogen el tema, dentro del campo de la asignatura que explican; otros acuden a sus personales aficiones, abordando aquellas cuestiones que particularmente han trabajado más o mejor conocen; y algunos presentan asuntos de novedad o de palpitante actualidad científica, aportando a la doctísima consideración del ilustrado auditorio que les escucha, la última palabra que la ciencia ha pronunciado sobre la cuestión, y que ellos se apresuran a poner sobre el tapete de la crítica; pero todos sin excepción, imbuidos de la idea de imprimir a sus composiciones y manifestaciones un sano espíritu de avance, procuran ofrecer el resultado de sus largas meditaciones, el fruto sazonado de hondas y pacientes investigaciones, agotando a este efecto cuantos recursos les sugiere su personal ingenio, y dejando a la vez entrever que han puesto en juego, al elaborar sus propias y particularísimas concepciones toda aquella habilitación de medios y de aptitudes de que han podido disponer y de que son capaces ${ }^{21}$.

Sin embargo, nosotros hemos entresacado mediante seguimiento al detalle, y su lectura en la mayoría de los casos, de un cupo importante de discursos académicos, pronunciados en la «fiesta de la ciencia» en diferentes universidades españolas, que sí contienen abundantes y sugerentes propuestas de mejora pedagógica y organizativa para su disciplina o especialidad y para la vida universitaria de su propio establecimiento, y también para la Universidad española en general. Esta laboriosa tarea nos ha permitido seleccionar 140 discursos académicos, pronunciados justamente el día de la inauguración del curso en las distintas universidades, que nos parecen una fuente de información de alto grado cualitativo.

21 Cfr. Murueta Goyena, Federico: Discurso leído en la Universidad de Valladolid en i9is (ver anexo), pp. 7-8. 
EL DISCURSO PEDAGÓGICO EN LA FIESTA DE LA CIENCIA EN LA UNIVERSIDAD DESEADA: ESPAÑA I9OO-I936 JOSÉ MARÍA HERNÁNDEZ DÍAZ

Para obtener la lectura de esta extraordinaria documentación ha sido preciso consultar varias fuentes digitales que facilitan el acceso (pensemos ahora, por ejemplo, en la Universidad de Granada), y de forma especial la Biblioteca Histórica de la Universidad de Salamanca, que conserva la mayoría de estas fuentes, de estos discursos.

Hemos elaborado un anexo a este trabajo, que ofrecemos, y da fe de todos los discursos que nos han servido de fuente documental directa de información, y que permiten al lector una aproximación subsidiaria al tema, y de comprobación y contraste si fuese necesario.

¿Qué tipo de discursos y propuestas merecen ser destacados?

En primer lugar hemos de indicar que la práctica más habitual en las universidades españolas de estos años (1900-1936), en relación al catedrático que ha de ofrecer el discurso inaugural en el día de la «fiesta de la ciencia», o también llamado día de la «fiesta de la labor intelectual» (ver discurso de Gómez Izquierdo, en la Universidad de Granada, I920), es que el orador sólo lo va a ser una vez en la vida, y así se respeta en la mayoría de los casos.

Pero no siempre es así. De hecho, en el listado que adjuntamos para poder confirmar y contrastar, aparecen ocho profesores que repiten dos discursos, si bien varios en universidades distintas. Así, Arias de Velasco y Lúgico, I9ıı, Oviedo y 1920, Oviedo; Casares Gil, 1900, Barcelona y 1922, Madrid; Hazañas y la Rúa, 1907, Sevilla y 1922, Sevilla; Miral López, 1908, Salamanca y 1917, Zaragoza; Onís Sánchez, I912, Oviedo y I920, Salamanca; Pareja Garrido, I9II, Granada y I922, Granada; Simonena Zabalegui, I905, Valladolid y 1923, Madrid; Unamuno, I900, Salamanca y 1934, Salamanca. Observamos que de estos ocho profesores, cuatro de ellos repiten en la misma universidad, curiosamente sobre asuntos de interés pedagógico. En la comprobación que hemos hecho del resto de discursos inaugurales (un total de 378 unidades documentales diferentes) de todas las universidades españolas, observamos que son solamente 24 del total de profesores los que repiten, incluidos los que aquí hemos seleccionado como de carácter pedagógico.

Aun a riesgo de cometer algún error de matiz en la clasificación, hemos seleccionado de ese total referido (378) un cupo de 140 discursos, que en nuestra opinión poseen elementos suficientes para ser considerados como pedagógicos, y que ocuparían el $37 \%$ del total. La asignación de esta categoría, el quedar o no incluido el discurso respectivo como pedagógico o no, la hemos atribuido en función de si contempla o no alguna de las siguientes ocho variables que ahora comentamos como elemento principal del tema que se expone retóricamente a la comunidad universitaria que le escucha. Tales indicadores nos van a permitir analizar si se están proponiendo cambios o novedades en búsqueda de una nueva universidad, que vayan construyendo la universidad deseada, o si nada más se trata de cumplir con el ritual, recibir los honores propios de la vanidad y ostentación intelectual que encierra un acto de esta naturaleza.

Los indicadores utilizados en la selección han sido los siguientes, expresados de forma esquemática y enumerativa, pues luego hemos de volver sobre cada uno de ellos «in extenso»: regeneración universitaria, misión y políticas generales de actuación; profesores y catedráticos; estudiantes; métodos de enseñanza y didácticas específicas de determinadas disciplinas; relaciones entre la universidad y la sociedad; 
relaciones expresadas con el sistema educativo previo a la universidad; cuestiones específicas de pedagogía científica; historia de algunas universidades. Vamos a ver a continuación en qué medida la muestra de discursos académicos seleccionados, pronunciados en la fiesta de la ciencia de alguna de las universidades públicas ya reseñadas, responde a cada una de esas sencillas categorías que hemos arbitrado. Para no reiterar en cada discurso la referencia completa, remitimos con los apellidos, año y universidad donde se pronuncia a la consulta del Anexo final.

\section{I. Regenerar la universidad española y convertirla para la ciencia}

El tema estrella que aparece en estos discursos de contenido educativo que hemos seleccionado del conjunto no puede ser otro que el de regenerar la universidad, la misión y función de la universidad, los males y remedios que han de corregirse en la universidad española, la política universitaria a impulsar, la posición ante la investigación y la modernización científica, la reorientación de los estudios, la presencia de la mujer en la universidad española como fenómeno inédito, el atraso de las ciencias experimentales en España, el debate sobre la autonomía universitaria, la reorganización técnica que precisa la universidad, las relaciones de proximidad que han de mantener las ciencias y sus cultivadores.

A estos temas los catedráticos oradores en los paraninfos dedican 40 discursos del total, y son los siguientes: Álvarez de Linera (La Laguna, I92I), Arias de Velasco (Oviedo, I9II), Banqué y Faliu (Barcelona, I9II), Bartolomé del Cerro (Valladolid, 1933), Bartual y Moret (Valencia, 1916), Calamita Álvarez (Zaragoza, I9II), Candau Pizarro (Sevilla, I9I2), Cañadas Domenech (Sevilla, I92I), Casares Gil (Barcelona, 1900), Comín Moya (Zaragoza, I9ı2), Deulofeu Poch (Santiago, I9I4), Díez Sánchez (Salamanca, I90I), Díez Tortosa (Granada, I919), Eleizegui López (1906), Fernández Baños (Santiago, 1929), Galcerán Cifuentes (Oviedo, I9I8), García Alas (Oviedo, I922), García García (Madrid, 1931), Gómez Izquierdo (Granada, 1920), Lasso de la Vega (Sevilla, 1904), Macher Vilar (Granada, 1903), Martín González (Madrid, I932), Melón (Valladolid, 1932), Miral López (Salamanca, I908), Onís Sánchez (Oviedo, I912), Pareja Garrido (Granada, I9II y I922), Pérez Bueno (Oviedo, 1905), Pérez Carballo (Madrid, I933), Román Retuerto (Salamanca, 1926), Rovira Carreró (Santiago, 1926), Royo González (Sevilla, 1919), Simonena (Madrid, I923), Testor Pascual (Valencia, I9II), Tormo Monzó (Madrid, 1909), Traviesas (Oviedo, 1914), Vijande (Oviedo, I917), Zabala Lera (Madrid, I919), Zumalacárregui y Prat (Valencia, 1919).

No vamos a exponer con detalle el contenido de cada uno de los discursos ahora mencionados, entre otras razones porque resultaría muy pesado para el lector reiterar argumentos muy parecidos entre sí. Nos referimos a la imagen, muy frecuente, de decadencia y crisis de la Universidad, acentuada por el clima de pesimismo social reinante después de la derrota de i898 en Cuba. Es lo que nos dice, entre varios más, Miral López en Salamanca en 1908. «En esta universal ruina que padece España no podía faltar la ruina de la universidad, cuyas raíces seculares están ya tan carcomidas que a duras penas pueden sostener en pie» ${ }^{22}$.

${ }_{22}$ Ver Anexo, Miral López, Salamanca, 1908, p. 7. 
EL DISCURSO PEDAGÓGICO EN LA FIESTA DE LA CIENCIA

EN LA UNIVERSIDAD DESEADA: ESPAÑA I9OO-I936

JOSÉ MARÍA HERNÁNDEZ DÍAZ

Igualmente, se alude a la responsabilidad de la Universidad en el proceso de regeneración de España, que ha de pasar por una regeneración intelectual y moral de la universidad. Para ello conviene mirar otras universidades extranjeras, como las alemanas, dice Casares Gil ${ }^{23}$, decano de la Facultad de Farmacia en Barcelona en 1900, sobre todo en la imagen de las ciencias experimentales.

La misión tradicional de formar especialistas para la administración y para las profesiones liberales es prioritaria de la actividad universitaria, comentan varios conferenciantes. Pero también, y ahora con más insistencia, emerge la función científica y creadora de la universidad, la extensión universitaria, la difusión de la ciencia y el impulso de la vida económica (Zumalacárregui y Prat, Valencia, 1919).

Persisten asuntos ideológicos entre las preocupaciones de los catedráticos, herencia del perverso sistema de control ejercido en el modelo de universidad del xIx, por lo que la libertad de enseñanza, la libertad de cátedra son asuntos no banales, que se hacen visibles en algunos de estos discursos (Comín Moya, Zaragoza, I9ı2; Díez Sánchez, Salamanca, I90I). También el debate sobre la autonomía universitaria en torno al decreto de Silió en 1919 (Zabala Lera, Madrid, I919).

Desear y alcanzar otra universidad mejor en España pasa por reorganizar las enseñanzas (Eleizegui, Santiago, 1906; García Alas, Oviedo, 1922; Gil Morte, Valencia, 1920; Macher Vilar, Granada, 1903; Vijande Fernández, Oviedo, 1917; Tormo Monzó, Madrid, I909; Simonena Zabalegui, Madrid, 1905); revisar y actualizar los planes de estudio (lo dice para medicina Pareja Garrido, Granada, I9ı y Roquero Martínez, Sevilla, 1909; y para derecho, Traviesas, Oviedo, 1914); introducir en la oferta de estudios a cursar nuevos campos científicos (Galcerán Cifuentes, Oviedo, I9I8); escuchar el momento político adecuado (García García, Madrid, I93I); actualizar el protocolo y las ceremonias universitarias (González Izquierdo, Granada, I920); facilitar la apertura y llegada de las mujeres a la universidad (Lasso de la Vega, Sevilla, 1904).

Surgen, en contrapartida, en algunos discursos voces menos pesimistas, hartas de escuchar hasta la saciedad los términos «regeneración» y «males de la patria» que los Picavea, Lucas Mallada, Julio Senador han difundido con énfasis y han calado en el imaginario colectivo de los españoles. Así sucede con Fernando Pérez Bueno, de la Universidad de Oviedo, quien en I90s escribe: «Digámoslo muy alto: el problema de nuestra enseñanza no es técnico, ni es científico, ni es doctrinal, ni es pedagógico; es, simplemente, cuestión de moral, de justicia y de higiene de las conciencias» ${ }^{24}$.

23 «La organización de la universidad alemana es admirable. Ha conseguido encontrar el medio de mantener un estímulo constante entre sus profesores, premiando como corresponde sus esfuerzos, y evitar un escalafón que si es cierto que pone al abrigo de graves injusticias mata también toda iniciativa. Con el método de su enseñanza y especialmente con sus estudios de doctorado inculca en el alumno el germen del progreso y de los nuevos descubrimientos. No debemos copiar todos los detalles de la organización de las universidades alemanas; pero sí imitar su espíritu» (ver Anexo, Casares Gil, Barcelona, 1900, p. 33).

La admiración también se percibe en la proyección científica exterior, por ejemplo de Alejandro Humboldt en América (Melón, Valladolid, 1932).

${ }^{24}$ Cfr. Anexo, Pérez Bueno, Oviedo, I905, p. 6. 
Al fin, se dejan oir voces sensatas, como la del catedrático de Ciencias de Salamanca Emilio Román Retuerto ${ }^{25}$, quien deja plasmada su aspiración sobre qué ha de hacer la universidad en España en la tercera década del siglo xx.

La Universidad, como agrupación de docentes y discentes, tiene por misión enseñar y cultivar todas las manifestaciones del espíritu humano; debe ser un taller en que se dilaten las inteligencias y se fortalezcan las voluntades; un foco donde se concentre la sabiduría y un órgano impulsor de la doctrina y de la educación nacional. La Universidad ha de proporcionar aquellos conocimientos que no dicen relación a la práctica, o sea los conocimientos especulativos, cuyo fin próximo es la contemplación de la verdad y que constituyen la entrada para las demás ciencias y facultades; ha de capacitar para el ejercicio de las distintas profesiones, que tengan por base las disciplinas especulativas; ha de educar para la investigación y ha de ejercer una misión social educadora.

Estas palabras muy bien nos pueden servir de síntesis apretada de lo que aspiran y desean un buen grupo de catedráticos, selectos universitarios, sin duda, sobre la institución que desean mejorar y enriquecer científica y humanamente.

\subsection{Una universidad diferente, cientifica y respetable precisa del buen profesor}

El segundo de los indicadores seleccionados en el muestrario de discursos inaugurales se refiere a los profesores, y en particular a los catedráticos, a quienes en el conjunto se dedica un escaso número de reflexiones, seis solamente. Nos referimos a los discursos de Bustos Miguel (Salamanca, I922), Cañizo García (Salamanca, 1927), Maldonado de Guevara y Fernández de Ocampo (Salamanca, 1919), Moliner Nicolás (Valencia, 1904), Párraga Acuña (Sevilla, 1907), Rivas Mateos (Madrid, 1912). Observemos algunas de sus reflexiones y propuestas que critican la situación de los profesores en la universidad española del momento, buscan y sueñan con otras circunstancias más favorables.

La intervención en 1904 del fogoso catedrático de Medicina de Valencia Moliner Nicolás, sobre la dimisión en la cátedra como elemento de protesta, quiere erigirse, a modo de inmolación, y «por conciencia profesional», en una llamada de atención sobre la lacerante situación que viven maestros, catedráticos y todos los docentes en escuelas y universidades, carentes de recursos para un ejercicio digno de su profesión ${ }^{26}$.

De manera más pausada y menos estridente que el anterior catedrático, en la Universidad de Madrid, en I9I2, el biólogo y catedrático de Farmacia Rivas Mateos habla sobre el profesorado universitario en un bello, lúcido y extenso discurso, en el que busca justificar las dos ramas de la actividad docente del profesor, la enseñanza de la ciencia a él encomendada y la educación de los jóvenes estudiantes que inseparablemente debe acompañarla. En su intervención expone

Cfr. Anexo, Román Retuerto, Salamanca, 1926, p. I7.

26 «Pues bien, señores profesores y alumnos: he aquí el momento psicológico y ético generador de mi determinación, de presentar la dimisión dentro de un par de meses si veo que los presupuestos de instrucción pública preparan para este año una enseñanza clínica como la del año anterior» ( $c f r$. Anexo, Moliner Nicolás, Valencia, 1904, p. 24). 
EL DISCURSO PEDAGÓGICO EN LA FIESTA DE LA CIENCIA

EN LA UNIVERSIDAD DESEADA: ESPAÑA I9OO-I936

JOSÉ MARÍA HERNÁNDEZ DÍAZ

su punto de vista sobre la situación del profesorado universitario en España y la posición que debe ocupar para el mejor cumplimiento de la misión docente, así como la organización que ha de tener el estamento que enseña. El profesorado ha de dedicarse con intensidad y exclusividad a la misión docente de la universidad, pero para ello precisa de independencia económica, y ser recompensado con un salario digno y reconocido a su posición y a la misión que la sociedad le tiene encomendada ${ }^{27}$. Pero el buen profesor universitario debe poseer también formación pedagógica adecuada, según nuestro orador, y en esto coincide plenamente con las propuestas de Giner y los institucionistas. «Parece imposible, nos dice, que la Universidad carezca de una escuela especial de Pedagogía para la preparación y formación del profesorado universitario». Y aplaude con fervor todo el proyecto de renovación que representa la Junta para Ampliación de Estudios a partir de 1907, reconociendo, a manera de ejemplo, que sus referentes organizativos como universidad son las de Alemania y Suecia.

Un discurso que podríamos considerar complementario del que acabamos de mencionar es el que pronuncia el catedrático de Ciencias de Salamanca, en I922, Bustos y Miguel. Es un sugerente texto pedagógico destinado a reflexionar sobre «la vocación para la enseñanza», y va dirigido a estudiantes y profesores, y en alguna de sus partes se dirige de forma expresa a los catedráticos, profesión tan especial y necesaria. Así expone en vivo «No temo indicar lo que yo exigiría para ser catedrático: conocimientos profundos de la asignatura, instrucción general y práctica docente, pero sobre todo, probada vocación $»^{28}$.

Agustín del Cañizo, catedrático de Medicina en Salamanca, en 1927 insiste desde la reflexión sobre modelos de profesores que él ha tenido en su vida en la responsabilidad moral del catedrático, en el valor de la ejemplaridad.

El discurso de Maldonado de Guevara en igro es un cuidado cántico a la excelencia de los grandes catedráticos y profesores, como fue el caso del penalista Pedro Dorado Montero en su cátedra de Salamanca, en esta ocasión plenamente merecido, sin excesivos alardes retóricos en el marco del paraninfo salmantino, tan llamado a determinados excesos. Finalmente, Párraga Acuña propone en Sevilla, 1907, que los cultivadores de las ciencias más diversas en el seno de la Universidad deben adoptar relaciones de intercambio y complementariedad, sin recluirse cada uno de ellos en su propia concha, en su cátedra.

\subsection{Exhortaciones a la juventud estudiante}

$\mathrm{Al}$ asignar título a este epígrafe destinado a los estudiantes nos inspiramos en el bello discurso que pronuncia Unamuno en Salamanca en I900, siendo catedrático de literatura griega, y que va dirigido expresamente a la juventud estudiosa.

27 «Si la situación económica de los catedráticos es a todas luces deficiente, ¿qué diremos de ese otro grupo de profesores, llamados auxiliares, que tan importante papel desempeñan en la vida y desenvolvimiento de las universidades? Esta clase, tan modesta y olvidada por los poderes públicos, y casi con el mismo rango social de los catedráticos numerarios, es caso de conciencia que, por honor de clase y hasta por sentimientos de humanidad, necesita urgente remedio. Dada su posición económica, no hay fuerza moral que obligue al exacto cumplimiento del deber» (cfr. Anexo, Rivas Mateos, Madrid, I9ı2, p. 35).

${ }_{28}$ Cfr. Anexo, Bustos Miguel, Salamanca, 1922, p. 3I. 
Son muchos los discursos inaugurales pronunciados que vagan retóricamente sobre el sentido del mismo y suelen dirigirse subsidiariamente a los estudiantes, pero en la práctica son muy pocos los que han pensado de manera directa y específica en los estudiantes que llegan por primera vez a las universidades, a sus aulas, y no saben qué es lo que van a hacer allí en realidad desde el punto de vista científico, más allá de obedecer las directrices de sus padres. Por ello, pensamos que de forma muy original e inteligente, el catedrático de Griego de Salamanca les invita a la creatividad, a la insatisfacción intelectual, a las preguntas, a empujar al profesor a que anime a los estudiantes y les inspire afición al estudio, a pensar en los problemas de la ciencia y de la vida cotidiana, a la curiosidad, a huir de la enseñanza libresca y de una educación con anteojeras. Solo así será posible una universidad diferente, aliada con la verdad y la ciencia, con la vida y los problemas reales que vive la gente. En ese contexto escribe también el poco más tarde «eterno» rector de Salamanca:

Los jóvenes que acudís hoy a estas aulas a que os traslademos lo que otros averiguaron, o lo que de la realidad hemos directamente averiguado nosotros, tenéis que interrogar a la realidad misma que se abre liberal a quien la invoca. Pero es preciso que la miréis cara a cara y sin interposición de librescos prejuicios, es menester que las lentes de las doctrinas recibidas no estén ahumadas adrede o por descuido. Las disciplinas que aquí se os transmiten son legado de los siglos, recordatorio de la humanidad, es cierto, pero también lo es, y con mayor plenitud aún, la realidad exterior concreta, la actualidad palpitante. En la vida común que os rodea, en las costumbres a que todos por hábito ajustamos nuestra conducta, en lo que sucede en la plaza, en el mercado, en la feria, en el templo, en el hogar o en la campiña late el pasado más vivo aun que en todos los libros, crónicas y documentos donde de ordinario no quedó más que su engañoso y deformado trasunto ${ }^{29}$.

Los estudiantes universitarios deberían ser los principales beneficiarios de este modelo de transmisión científica y académica que representa la lectio de la fiesta de la ciencia, que como ya hemos indicado mantiene evidentes resonancias medievales. A este capítulo de los estudiantes se dedican en conjunto pocos discursos, solamente ocho, pero algunos de ellos extremadamente bellos, como vemos a continuación: Gil Fagoaga (Madrid, I929), López Prieto (Valladolid, 193i), Rodríguez Pinilla (Salamanca, I913), Sesé Villanueva (Salamanca, r9ro), Simonena Zabalegui (Valladolid, 1905), Torres Campos (Granada, 1907), Unamuno (Salamanca, I900 y Salamanca, 1934).

Nicolás de Otto Escudero desde la Universidad de Murcia, en 1929, justifica con no exenta enjundia y elocuencia, la importancia del acto solemne de apertura del curso, y en particular la atención que merecen los estudiantes en el mismo. Éstas son sus palabras:

De igual modo que en las familias se celebra con júbilo y fiestas extraordinarias, con alegría y regocijo grandes, el advenimiento de nuevos vástagos, la Universidad, siempre acogedora, abre de par en par sus puertas a los noveles alumnos que van a integrar su población en lo sucesivo. En esto la universidad española tiene una limpia y acreditada ejecutoria, por cuanto desde las antiguas universidades mayores y

29 Cfr. Anexo, Unamuno, 1900, Salamanca, p. 7. 
EL DISCURSO PEDAGÓGICO EN LA FIESTA DE LA CIENCIA

EN LA UNIVERSIDAD DESEADA: ESPAÑA I9OO-I936

JOSÉ MARÍA HERNÁNDEZ DÍAZ

menores ha dispensado, con procedimientos y usos paternalmente democráticos, una acogida cordial a los estudiantes de todas las clases sociales ${ }^{30}$.

Ampulosas declaraciones retóricas como la precedente suelen dirigirse en los paraninfos a los estudiantes, en algún momento del discurso por parte de muchos oradores. Son peroratas que suelen carecer de sentido práctico y científico, muy alejadas, por ejemplo, del breve pero enjundioso discurso de Unamuno que ya hemos comentado, y que busca atribuir un nuevo significado a la participación de los jóvenes estudiantes en la construcción de una universidad más científica y anclada en la realidad y sus problemas. Muchos años después, en I934, en su despedida como profesor al jubilarse, Unamuno desgrana otro precioso y sugerente discurso sobre «la palabra», que invita a cultivar a los estudiantes, porque es el corazón de la cultura y de la ciencia; «se conquista con la palabra», dice al final de su intervención.

Pero también encontramos en diferentes universidades españolas aportaciones elocuentes que son acordes con el sentido del acto que se celebra en la inauguración del curso, y que se orientan específicamente a los estudiantes. Unas, como las de Gil Fagoaga (Madrid en 1929) y López Prieto (Universidad de Valladolid en 1931), inciden en asuntos muy particulares, como son los relativos al sistema de selección profesional de los estudiantes, y en concreto los de Medicina, buscando que su formación, vocación y estímulos sean los más adecuados a la posterior profesión de médico, y no se desaprovechen esfuerzos y medios ante estudiantes desmotivados y sin condiciones. Dos catedráticos de Salamanca, Rodríguez Pinilla (Medicina, en I913) y Sesé Villanueva (Ciencias, en I9ıo), así como Simonena Zabalegui en Valladolid (1905), inciden expresamente sobre la importancia de la dedicación intensiva al estudio y de encontrar los métodos más apropiados para ser exitosos en el esfuerzo prestado a la actividad formativa. Rodríguez Pinilla menciona de forma muy oportuna la conveniencia de que la universidad española fuera capaz de incorporar la tutoría de forma expresa, como ocurre en Inglaterra, donde el alumno cuenta con el apoyo y orientación de un profesor desde que ingresa en la universidad ${ }^{31}$.

Desde el punto de vista del estudiante, sus demandas asociativas para cubrir necesidades materiales, de acogida, de celebración y disfrute es muy interesante el texto que presenta en Granada (en 1907) el catedrático de Derecho Torres Campos. No habla en ningún momento de la universidad española, pero sí lo hace de forma muy documentada en términos históricos comparados de los diferentes modelos asociativos para los estudiantes que pueden ser observados ( $\mathrm{y}$ seguramente imitados o al menos considerados en España) en Francia, Alemania, Inglaterra, USA, Rusia, Suecia, en todo el mundo avanzado a comienzos del siglo $\mathrm{xx}$ en asuntos universitarios. Este autor, de clara vinculación con el espíritu de la ILE, añora en España, al inicio de su intervención, un cambio profundo en la organización de nuestra Universidad y enseñanza en general ${ }^{32}$.

3 Cfr. Anexo, Otto Escudero, Murcia, I928, p. 9.

${ }_{31}$ «Si en nuestro país existiese, como en Inglaterra, lo que se llama el tutor de estudios, alguien distinto del profesor, que se encargase de dirigir las tendencias del escolar, además de pasarle las lecciones, encauzarle por el derrotero que inicia el niño mismo, el niño motor o sensitivo, las vocaciones serían más numerosas y precoces» (ver Anexo, Rodríguez Pinilla, Salamanca, I913).

${ }_{32}$ "Continuamos esperando en España a un nuevo Mesías que, como el ilustre Ferry en Francia, transforme por completo los establecimientos docentes en sentido progresivo» (Anexo, Torres Campos, Granada, 1907, p. 3). 


\subsection{Nuevos métodos de enseñanza para una universidad moderna}

La atención a la reforma de los métodos de enseñanza utilizados en la universidad, y en particular a la didáctica de algunos campos disciplinares, representa uno de los indicadores de mayor atractivo para los oradores que intervienen en los paraninfos en este día tan especial de la inauguración del curso en cada universidad española. Es un reflejo evidente del deseo de ofrecer en las aulas una enseñanza renovada, de buscar otro tipo más activo de docencia, de mejorar y ser más eficaces. Por ello nos encontramos que un total de 39 discursos académicos se centran en esta parcela didáctica de la reflexión universitaria, representando un $28 \%$ del total de los estudiados. Vamos a ver la diversidad de ciencias y campos disciplinares que se desea renovar, así como quiénes son sus proponentes.

Nos situamos ante un cualificado y abundante elenco de catedráticos deseosos de introducir novedades en su docencia del derecho, la medicina, química, biología, matemáticas, física, farmacia, historia natural, geografía, historia, la lógica y la filosofía, lenguas semíticas, derecho mercantil, disciplinas estéticas y otras: Andrés Irueste (Madrid, I9Io), Apráiz Buesa (Barcelona, 193I), Barras y de Aragón (Oviedo, 1907), Buén (Barcelona, 1909), Casamada Maurí (Barcelona, 1930), Casares Gil (Madrid, I922), Castell Miralles (Valencia, 1905), Castro y de Castro (Sevilla, 1902), Deleito Piñuela (Valencia, I9I8), Domenech Estapá (Barcelona, 1904), Espurz Camobarde (Oviedo, 1909), Fresno y Pérez del Villar (Oviedo, 1930), García Varela (Santiago, I9II), Garrido Osorio (Granada, I90o), Goizueta Díaz (Barcelona, 1915), Gonzalvo París (Valencia, 1914), Izquierdo Gómez (Valencia, I917), Jiménez de Cisneros (La Laguna, 1924), López Capdepón (Barcelona, 1920), Madinaveitia Tabuyo (Madrid, 1927), Meneu Meneu (Salamanca, 1916), Mollá Rodrigo (Madrid, 1928), Mur Ainsa (Oviedo, 1910), Nacle Herrera (Granada, 1930), Novoa López (Santiago, 1904), Olóriz Ortega (Granada, 1927), Pastor Pellicer (Zaragoza, 1902), Población Sánchez (Salamanca, I93i), Puente Sánchez (Santiago, I922), Requejo Alonso (Salamanca, I9II), Rodrigo Lavín (Sevilla, I926), Rodríguez Ávila (Granada, 1909), Rodríguez Fernández (Madrid, 1913), Rodríguez Risueño (Valladolid, I90I), Roquero Martínez (Sevilla, I909), Soriano Sánchez (Barcelona, 1906), Torremocha Téllez (Valladolid, 1927), Ureña y Smenjaud (Madrid, 1906), Vilariño Magdalena (Santiago, 1909).

Sin poder entrar ahora en el estudio pormenorizado de cada una de las propuestas de reforma pedagógica y didáctica en las diferentes asignaturas o campos disciplinares que aparecen en este cupo de discursos solemnes (lo que bien podría trasladarse al estudio de la historia de las disciplinas, como puede derivarse de sugerencias como las de Chervel ${ }^{33}$ o Antonio Viñao), es de interés mencionar algunas vías de investigación y estudio que se derivan de ellos.

Así, para mejorar la universidad, y sobre todo la imagen y eficacia docente en la respectiva disciplina, los catedráticos ponen su énfasis particular en las dificultades materiales y económicas que existen para una adecuada dotación

33 Cfr. Chervel, André: «Historia de las disciplinas escolares. Reflexiones sobre un campo de investigación», Revista de Educación, Madrid, 295 (199I), pp. 59-III; VIÑAO, Antonio: «La historia de las disciplinas escolares», Historia de la Educación, Salamanca, 25 (2006), pp. 243-269. 
EL DISCURSO PEDAGÓGICO EN LA FIESTA DE LA CIENCIA

EN LA UNIVERSIDAD DESEADA: ESPAÑA I9OO-I936

JOSÉ MARÍA HERNÁNDEZ DÍAZ

y actualización de bibliotecas y laboratorios. Las ciencias experimentales, la medicina, la farmacia parecen adolecer, en boca de sus catedráticos, de condiciones suficientes para poner en marcha una docencia práctica y experimental correcta. Las humanidades y ciencias sociales precisan de más medios, de más y mejores revistas, buenas y bien dotadas bibliotecas, de otros apoyos concretos de carácter material, sin mencionar para nada aquí asuntos como los salarios, tipología y número de profesores, entre otros. Todas las ciencias, sin excepción, reclaman más presupuesto, más atención y sensibilidad por parte de los responsables del Ministerio de Instrucción Pública, de quien todas las universidades dependen.

Pero en los discursos emergen también otras posiciones y reclamos de orden organizativo y epistemológico, relativas a la inadecuada organización de los estudios en las facultades, a la desconexión entre las distintas ciencias, y de la necesidad de planificar con urgencia una lectura interdisciplinar del conocimiento, de las ciencias y de su enseñanza. En todo este planteamiento afloran experiencias didácticas novedosas, procedentes de países más avanzados en la organización y docencia universitaria, de Europa y de Estados Unidos. Es frecuente la mención a la JAE en estos discursos, una vez que se han iniciado los programas de pensionados a partir de 1907 .

\subsection{La universidad se debe a la sociedad}

Otro de los capítulos que interesa mucho a los oradores de los paraninfos españoles en esta etapa de la historia universitaria es el relativo a las relaciones entre la Universidad y la sociedad, como misión social, relación con la industria y las diferentes profesiones emergentes, proyección internacional de la lengua española y de la Universidad, entre otros capítulos y elementos.

Nos encontramos, después de la selección del elenco, con un número de 17 discursos que se proyectan en estas dirección: Aranda Millán (Zaragoza, 1931), Aranzadi Unamuno (Barcelona, I905), Borobio Díaz (Zaragoza, I910), Candela $\mathrm{Pla}$ (Valencia, 1900), Coll Pujol (Barcelona, 1913), Cuenca Araujo (Santiago, 1918), Díez Canseco (Valladolid, I908), Esperabé de Arteaga (Salamanca, 1924), Fernández del Riego (Santiago, 1933), Giral Pereira (Salamanca, 1918), Iranzo (Zaragoza, 1914), Lozano Rey (Madrid, I915), Onís Sánchez ( Salamanca, I920), Otto Escudero (Murcia, I928), Peset y Cervera (Valencia, 1924), Ríos Urruti (Granada, 1917), Sánchez Gallego (Murcia, 1919), Valentí Vivo (Barcelona, 1903).

La primera de las funciones que la universidad ha de cumplir con la sociedad es la formación de buenos profesionales (Díez Canseco, Valladolid, 1908), sean abogados (Sánchez Gallego, Murcia, 1919), farmacéuticos (Cuenca Araujo, Santiago, 1918), profesores, médicos (Iranzo, Zaragoza, 1914). Pero también ha de servir a los intereses de su sociedad más próxima (en este caso Galicia, Fernández del Riego, Santiago, 1933),

Es frecuente escuchar en estos discursos que la Universidad está muy desconectada de la sociedad, pues ostenta un permanente monólogo, desesperante para los intereses de la población, como dice el catedrático de Derecho de la 
EL DISCURSO PEDAGÓGICO EN LA FIESTA DE LA CIENCIA

EN LA UNIVERSIDAD DESEADA: ESPAÑA I9OO-I936

JOSÉ MARÍA HERNÁNDEZ DÍAZ

Universidad de Murcia en 1919, Mariano Sánchez Gallego ${ }^{34}$. Otro catedrático de Medicina, Rivas Mateos, desde la Universidad de Madrid incide en I9I2 en esta cuestión del alejamiento de los intereses reales del pueblo y la necesidad de buscar fórmulas de aproximación ${ }^{35}$.

El discurso de Valentí Vivo en la Universidad de Barcelona (1903), de decidida orientación hacia la fraternidad universal, caminando entre el positivismo y el pansofismo, atribuye a la ciencia y a la función influyente de las minorías una tarea irrenunciable para el bienestar, la armonía y el progreso de los pueblos. Así nos dice, «progresan los pueblos por armónico esfuerzo de inteligencias superiores que se proponen el bien, la salud, la verdad, el progreso, la justicia y laboran en pro de estos máximos ideales con actos de razón y experiencia colectivas» ${ }^{36}$. Desde otra lectura, Fernando de los Ríos Urruti (Granada, 1917), quien desempeñará en la Segunda República la cartera de ministro de Instrucción Pública, de forma indirecta reflexiona sobre la necesaria apuesta por estructuras democráticas en el Estado y en la sociedad, incluidas las instituciones docentes.

Pero encontramos también posiciones muy reticentes a la apertura de la universidad española hacia la ciencia y los modelos procedentes del exterior, y en concreto hacia Europa. Así, Borobio Díaz, catedrático de Medicina en Zaragoza (1910), afirma que en la universidad hay que hacer ciencia y hacer patria, y dice con contundencia que no le gusta lo de europeizar España ${ }^{37}$, y lo argumenta en

34 «Hoy, señores, la Universidad, sea cual sea la causa, marcha en sentido divergente de la sociedad; cada paso de su vida es un distanciarse del pueblo; Universidad y pueblo hablan un idioma distinto; miran a horizontes distintos; ayer la Universidad era el corazón poderoso que volcaba el torrente de sangre cálida sobre las sociedades, para que vivieran del sobrante de vida universitaria; hoy la Universidad es el corazón apagado que necesita para latir de inyecciones tonificantes gubernativas y de aires que corran de fuera hacia la Universidad. Por eso, señores, cuando en días como hoy he oído discursos bellísimos sobre temas de profunda investigación, me han parecido un monólogo sublime y desesperante en el vacío de un desierto; una melodía fantástica que sonara durante la noche en el silencio de un cementerio; una exposición de colorido ante una sociedad a quien cegó el Destino; era Pierrot que contaba sus sueños hermosos a quien no le interesaban los sueños de Pierrot» (cfr. Anexo, Sánchez Gallego, Murcia, 1919, p. 7).

35 «Hay que empezar arrancando la universidad de ese encastillamiento medieval que más invita a la meditación sobre la buena muerte que al encanto de la hermosa vida; hay que llevarla a la calle, al pueblo, para que luche y se remoce, que suelte la modorra y tome bríos y aires de afuera; y no importa que al chocar con la rudeza ineducada se contamine con el error, que eso pasa en la Universidad de Londres y en todos los centros universitarios populares; al cabo, ello es motivo de lucha y la verdad vuelve a imponerse. El médico que cuida a un tífico es el más expuesto a infestarse, pero nadie me negará que es el que salva a los atacados, arrebatando vidas a la muerte. La universidad mezclada con el pueblo no desciende, se eleva; como desciende es dando patente de útil a los que están inservibles para la lucha y para el cumplimiento del deber. Y esto lo ven y lo saben nuestros conciudadanos, y esto es ¡hay que decirlo!, un producto de fábrica que desacredita la marca de tal manera que en el mercado ofrecen por él poco valor» ( $c f r$. Anexo, Rivas Mateos, Madrid, I9I2).

${ }_{36}$ Ver Anexo, Valentí Vivo, Barcelona, I903, p. I9.

37 «Se habla de europeización. Me es antipática la palabra, neologismo metido a cuña en la lengua de Cervantes por un cerebro poderoso, en días de gran desaliento nacional; y en cuanto a su significación, entendámonos. Si por europeizar se entiende olvidar nuestra historia, despreciar nuestro arte, censurar nuestro derecho, costumbres e instituciones, desconocer nuestro suelo, borrar quizá nuestras fronteras, anular nuestra raza y nuestra persona, abominar, en fin, de todo lo propio y preferir todo lo ajeno, secarse han, sin conseguirlo, las manos que en tan vitando empeño se pongan: no se extingue un pueblo por una momentánea tendencia político-social. Para la europeización en el 
EL DISCURSO PEDAGÓGICO EN LA FIESTA DE LA CIENCIA

EN LA UNIVERSIDAD DESEADA: ESPAÑA I9OO-I936

JOSÉ MARÍA HERNÁNDEZ DÍAZ

un discurso pesimista sobre la Universidad española que, dice él, está sometida al formalismo del Estado y carente de la autonomía y libertad, que debieran ser los rasgos de una universidad regida por los catedráticos.

La atención a la industria desde la Universidad es una de las funciones prácticas y con proyecto social y económico claro, que comienza a advertirse en la España que se abre a la industrialización de forma más sólida, después del desastre del 98. La universidad ha de contribuir a mejorar o construir el tejido industrial de España. Así lo observaba en I9ı8, desde la Universidad de Salamanca, el catedrático de Química José Giral, quien en un certero discurso dice, entre otras propuestas, «Si la ciencia es la impulsora del movimiento industrial y la universidad es, o debe ser, el organismo en donde se asienta su función, la consecuencia inmediata es que, para el fomento de la industria, se hace preciso ayudar a la universidad, o a los universitarios, en su labor ${ }^{3^{8}}$.

Cultivar el español con proyección internacional, de forma pionera en los Estados Unidos, ha sido una reflexión lúcida de Onís Sánchez (Salamanca, 1920). Quien ocupó la primera cátedra de Español y cultura española en la Universidad de Columbia, y en toda la historia universitaria estadounidense, afirma sin reparos que la falta de vida internacional es uno de los graves defectos que padece la Universidad española, y debe ser subsanado progresivamente. De ahí el aplauso que concede a la política de fomento de envío de jóvenes profesores e investigadores a las universidades extranjeras impulsada por la JAE desde 1907, de la que él mismo fue pensionado beneficiario. Desde otra perspectiva, se anima a los universitarios a proyectar la rica cultura española en la mundial, tal como expone Peset Cervera en un documentado discurso pronunciado en la Universidad de Valencia en 1924.

Son muchas las facetas relacionadas con la proyección necesaria de la universidad en la sociedad que hemos tocado, porque así aparecen en el registro de discursos inaugurales que hemos realizado. Sin duda quedan otras por desvelar. Volviendo al comienzo, y siguiendo parte del discurso de Esperabé de Arteaga (Salamanca, I924), la función social principal que ha desempeñar la universidad es la de ser el espacio de la alta cultura, de cultivo de la ciencia,

sentido científico, el modelo propuesto por los europeizadores no será seguramente Rusia, ni Turquía, ni Bulgaria, Serbia, Rumanía, Montenegro, Grecia, Suecia ni Noruega, aunque son países de Europa. Cuando se piensa en europeizar, la intención se fija, con variantes de motivo, en Alemania, Inglaterra, Francia, en primer término; en segundo, Bélgica, Holanda, Suiza e Italia, naciones que llevan en sí lo más perfecto del espíritu europeo. ¿Cómo han ganado ellas el puesto delantero en progreso y cultura? ¿Mirando hacia fuera? ¿Imitando al vecino? Nada de eso. Mirando hacia dentro, fomentando su agricultura y su industria, mejorando su enseñanza, sus leyes, su ejército, haciendo una intensa vida interior; formando su peculiar carácter, trazándose una dirección fija en su finalidad como nación; así, nacionalizándose, se han europeizado. Este es el camino. Conservar lo nuestro, mejorándolo, cultivar nuestras propias energías, multiplicar nuestra riqueza, edificar el porvenir sobre los cimientos del pasado, adaptar a nuestra raza lo bueno que tienen otras, modelar nuestro carácter, tonificar el alma nacional, robusteciendo el sentimiento, hoy decaído, del propio valer, no importar ciencia hecha, sino medios y modos de fabricarla aquí original, como árbol frondoso que tome la savia de nuestro suelo y la luz de nuestro cielo. Si se dice que esto es europeizar, yo digo que es españolizar, hacer ciencia y hacer patria. ¡Bendita ciencia que sostienes a la patria, y bendita patria que cobijas a la ciencia». Cfr. Anexo, Borobio y Díaz, Zaragoza, I9ıo, pp. 46-47.

${ }_{38}$ Ver Anexo, Giral, Salamanca, 1917, pp. 6o-6I. 
donde se va a enseñar y aprender, pero para ponerse al servicio de toda la sociedad, formando profesionales en diferentes especialidades y cultivando las ciencias con un sentido universal.

\subsection{La universidad y el sistema educativo deben entenderse}

De vez en cuando, de forma aislada, algún académico tiene presente en su reflexión al conjunto del sistema educativo, a los niveles escolares previos a la Universidad y al conjunto del mismo, buscando un adecuado ensamblaje para eliminar o mitigar la descoordinación que se observa con frecuencia. Esto es lo que, en este subapartado indicador, incluimos con nueve discursos solemnes de indudable interés, que se pronunciaron en esa etapa en las universidades sobre el tema general mencionado, sea la escuela infantil, la formación profesional y otros asuntos. Así encontramos las intervenciones de Arias de Velasco y Lúgigo (Oviedo, 1920), Bonet Bonet (Madrid, 1907), Canella Secades (Oviedo, 1913), Cerrada Martín (Zaragoza, 1918), Frontera Aurrecoechea (Oviedo, I915), Lázaro Ibiza (Madrid, 1902), López R. Gómez (Valladolid, I906), Segovia Corrales (Salamanca, 1909).

De la lectura de estos documentos entresacamos que la preocupación de los académicos por los niveles previos del sistema educativo es relativa, cuando no escasa en su conjunto. Ello a pesar de que, como es bien sabido, el sistema educativo español, siguiendo pautas francesas, que ya hemos comentado, atribuye a la universidad de orientación napoleónica una función administrativa directiva y jerárquica, de vigilancia y control sobre la escuela primaria y secundaria, sobre todos los establecimientos de enseñanza de su distrito (el rector es la máxima autoridad), aunque también tiene asignada otra de carácter orientativo y organizativo sobre los escalones inferiores, algo que suele brillar por su ausencia. Esto también puede observarse, en nuestra opinión, en la escasa apreciación que merecen los temas no universitarios en el seno de la institución superior, y no solamente en este muestreo de discursos. De ahí que, analizando el contenido de estos textos de retórica universitaria, que a la vez son expresiones documentales de un periodo histórico tan preciso, entendamos que adopten carácter testimonial la reflexión sobre la enseñanza en el distrito de Oviedo, alguna propuesta de mejora sobre la segunda enseñanza en España (es frecuente argüir con mucha retórica a algunos catedráticos que la universidad no puede resolver en los estudiantes las carencias que proceden de la segunda enseñanza), la educación infantil y otras pocas más.

De especial significado nos parece la propuesta de instaurar en España la formación profesional, que sugiere en igis José María Frontera Aurrecoechea, de la Universidad de Oviedo. Este catedrático de Matemáticas, que conoce bien el sistema educativo alemán que en su día diseñó G. von Humboldt, expone que la universidad no puede recluirse en sus claustros, sino que debe orientar la vida económica, social y la formación de quienes han de ocupar responsabilidades profesionales en la agricultura, la industria, el comercio o la administración pública. Para ello deben seguirse las pautas de los pedagogos alemanes, que han resultado exitosas, en la organización de cursos técnicos y la redacción de 
EL DISCURSO PEDAGÓGICO EN LA FIESTA DE LA CIENCIA

EN LA UNIVERSIDAD DESEADA: ESPAÑA I9OO-I936

JOSÉ MARÍA HERNÁNDEZ DÍAZ

programas profesionales especializados. Solo de esa manera España saldrá del triste letargo en que se encuentra ${ }^{39}$.

Como asunto manido, pero imprescindible, aparece mencionado el clásico binomio salvador de Joaquín Costa, «despensa y escuela», o el unamuniano «ciencia y carne», como elementos imprescindibles de la regeneración de los españoles y de la patria, que también precisa de una educación ciudadana muy mejorada, como propone Nicolás López R. Gómez en 1906, en el paraninfo de la Universidad de Valladolid.

\subsection{Apuntes de pedagogía científica}

Tal vez sea atrevido avanzar estos apuntes de pedagogía científica que puedan derivarse de algunos discursos académicos pronunciados en día tan solemne y señalado de la vida universitaria española de principios del siglo xx. Más aún cuando sabemos que hasta 1932 la pedagogía no alcanza rango universitario y profesional en España, y que solo en igos ha sido creada la primera cátedra de Pedagogía, ocupada por Manuel Bartolomé Cossío. Los loables esfuerzos sobre investigación pedagógica desarrollados desde el Museo Pedagógico (nacido en I882), desde profesores y ámbitos de influencia de la Escuela Superior de Estudios del Magisterio (desde 1909) y a partir del apoyo que desde I907 la JAE presta a algunos profesores jóvenes e inspectores son parte de un proceso incipiente de elaboración de las primeras aportaciones propiamente científicas producidas en España en el campo pedagógico. Ello explica que algunas investigaciones pedagógicas aplicadas a la escuela como institución en España fueran elaboradas, de forma aislada y testimonial, por catedráticos procedentes de la medicina, las ciencias experimentales o el derecho. Eso es lo que refleja el breve elenco de testimonios que ahora comentamos.

Son discursos académicos que se centran en cuestiones de pedagogía científica, aunque en un escalón muy inicial todavía, dada la bisoñez que ofrece una ciencia tan joven en su desarrollo como es la pedagogía. No deja tener su interés observar que comienzan a surgir preocupaciones por la mejora del sistema educativo, pero no de forma genérica solamente, sino también con propuestas y estudios anclados en la investigación. Así lo observamos en los diez discursos pronunciados en diferentes universidades españolas en este primer tercio del siglo xx, por Berenguer Ballester (Barcelona, I919), González Meneses (Sevilla, 1935), Ledo Eguiarte (Valladolid, I903), Miral López (Zaragoza, 1917), Murueta Goyena (Valladolid, I915), Pérez Martín (Oviedo, 1904), Piñerúa

39 «Hora es de que nuestra nación despierte del letargo en que vive sumida desde largo tiempo. Hoy que, a diferencia de antaño, ya no es fuerte porque no se cotizan aquellas empresas heróicas y atrevidas aventuras, hoy que se tiene en poco el valor personal si no lleva por armas las conquistas de la ciencia; en el siglo del dirigible y del submarino, no puede, no debe España quedar rezagada en medio siglo. Se hace precisa una profunda reacción y a ella debemos contribuir todos, hasta conseguir que nuestra nacionalidad sea respetada; hasta lograr desaparezca para los demás países el dudoso concepto que les merecemos, hasta borrar en suma la molesta frase de pobre español, que tan falta de compasión como sobrada de ironía asoma envuelta en una despreciativa sonrisa a los labios del extranjero que nos contempla» (ver Anexo, Frontera, Oviedo, I9I5, p. 58). 
EL DISCURSO PEDAGÓGICO EN LA FIESTA DE LA CIENCIA

EN LA UNIVERSIDAD DESEADA: ESPAÑA I9OO-I936

JOSÉ MARÍA HERNÁNDEZ DÍAZ

Álvarez (Madrid, 1920), Santos Fernández (Valladolid, I909), Simonena Zabalegui (Valladolid, I905) y Vargas Martínez (Barcelona, I918).

La demanda de una educación moral, la apuesta por una educación con valores ciudadanos la encontramos en los discursos de Berenguer Ballester, Pérez Martín, Piñerúa Álvarez y Vargas Martínez. Desde la medicina es de interés subrayar la atención que se presta a los problemas de la infancia, como la mortalidad infantil (así González Meneses), las enfermedades escolares (Santos Fernández) ${ }^{40}$ o la organización adecuada del estudio (Simonena Zabalegui). Existe algún catedrático, como Miral López, en Zaragoza, que en 1917 hace apuestas por la identidad regional por medio de la educación, en este caso por una pedagogía aragonesa, seguramente en el contexto de emergencia de diferentes regionalismos en España. Finalmente, un estudio histórico y comparado, de llamada de atención, es el que pronuncia en Valladolid Murueta Goyena, catedrático de Medicina, sobre las bondades de la escuela de los EE. UU. y su comparación con la escuela de países europeos, desde luego la española.

Nos parece de gran interés poder observar en este muestrario en qué medida las aportaciones procedentes del campo de la medicina forman parte de los preludios de la pedagogía científica, coincidiendo en que las profesiones de maestro y médico son profesiones sociales, y en buena medida vocacionales, y que los estudios de paidología se encuentran en estas fechas en una fase de elevado reconocimiento en la comunidad científica. Ello nos recuerda mucho a figuras estelares de la pedagogía contemporánea, como Claparède o Montessori (médicos también), o desde otro campo profesional el mismo Domingo Barnés ${ }^{4}$.

\subsection{La historia gloriosa de la vieja universidad española sigue viva}

La última de las categorías utilizadas en nuestro análisis de los discursos académicos es la que recoge los diez testimonios expuestos en los paraninfos, dedicados en estos años a honrar o legitimar la historia gloriosa de una universidad concreta o de un profesor excepcional de la misma, en particular en las etapas

40 Este catedrático de Medicina ofrece un precioso trabajo sobre los edificios escolares, el alumno y la inspección médico-escolar, de enorme actualidad en ese momento de la historia de España, cuando la alimentación infantil era deficitaria, muchas escuelas eran construcciones infames y antihigiénicas, y reinaba el descontrol en la administración escolar de España. Así, entre otras muchas sugerencias, nos dice: «La condición primera de toda buena inspección, es que esta sea una inspección verdadera para que merezca tal nombre, es decir, continuada, frecuente y sin interrupciones, llevada a feliz término por un personal de reconocida aptitud, y lo que es preferible, de una honorabilidad y rectitud completa, para que inspire confianza y seguridad en que sabrá cumplir los altos y trascendentales fines que se les confían, como es la preciosa e inapreciable salud de nuestra población infantil» (vid. Anexo, Santos Fernández, Valladolid, 1909, p. 82).

${ }^{41}$ Carmen Sanchidrián ha estudiado en profundidad la aportación de Montessori. Uno de sus últimos trabajos, «Montessori en España» se encuentra en vías de publicación en Ediciones Universidad de Salamanca, dentro del libro La pedagogía italiana en España e Iberoamérica. Respecto a Eduardo Claparéde, remitimos a nuestro estudio, «La educación en la obra de Eduardo Claparède», en Vergara Ciordia, Javier et al. (coords.): Ideales de formación en la Historia de la Educación, Madrid, Dykinson, 20II, pp. 437-457. En relación a Domingo Barnés, Hernández Díaz, José María: Paidología. De Domingo Barnés. Edición y estudio introductorio, Madrid, Biblioteca Nueva, 2008. 
EL DISCURSO PEDAGÓGICO EN LA FIESTA DE LA CIENCIA

EN LA UNIVERSIDAD DESEADA: ESPAÑA I9OO-I936

JOSÉ MARÍA HERNÁNDEZ DÍAZ

más gloriosas de su historia. Así sucede con los discursos de Bonilla San Martín (Madrid, 1914), Cabeza de León (Santiago, 1917), González de la Calle (Salamanca, 1912), Hazañas y la Rúa (Sevilla, 1907 y 1922), Loustau (Murcia, 192I), Maldonado (Salamanca, I933), Martos de la Fuente (Granada, I928), Riba García (Valencia, I9II, y Zaragoza, 1933), Serra Rafols (La Laguna, I93I), Téllez de Meneses (Salamanca, 1925), Torre del Cerro (Barcelona, 1926).

Desde la Universidad de Madrid, en igr4 Bonilla y San Martín habla de la universidad clásica y sus tradiciones perdidas como consecuencia de la aplicación del modelo uniformizante de los liberales, «que ha convertido a la universidad en una oficina más» del aparato administrativo de la enseñanza oficial, de un Estado que tutela y controla ${ }^{42}$. Esta intervención del catedrático madrileño es un cántico a la excelencia del modelo tradicional de universidad, anterior a las reformas ilustradas y liberales, y un alegato contra lo que considera un intervencionismo del Estado sobre la autonomía universitaria, diríamos nosotros, de sabor más rancio y anacrónico. Por ello, la lectura que hace de propuestas como las de Giner, o las específicas de la Junta para Ampliación de Estudios, expresa comentarios indolentes y escépticos sobre el modelo renovador de universidad que propugnan, sobre todo por el carácter estatalista e intervencionista que en su opinión representan en detrimento de la vieja autonomía universitaria todavía anhelada por este sector de catredráticos.

Otros discursos inaugurales próximos en temática relativa a las universidades del Antiguo Régimen, no siempre de parecidas posiciones ideológicas, buscan rescatar formas organizativas de la universidad medieval (caso de Lérida, en Serra Rafols, La Laguna, 193I); recordar fórmulas de selección de cátedras (como en Barcelona en el siglo XVI), como postula Torre del Cerro en 1926 en la Universidad de Barcelona; añorar estilos de vida universitaria en la Sevilla de la etapa moderna (así lo postula Hazañas en su universidad sevillana en 1907 y 1922), en la de Salamanca renacentista (Maldonado de Guevara en 1933), en la de Santiago de Compostela (Cabeza de León, 1917) o en el conjunto de las clásicas en la etapa precedente a las reformas ilustradas, como propone en Granada el catedrático Martos de la Fuente en 1928.

Algunos discursos enfatizan personalidades brillantes de aquellos siglos, como es el ejemplo de Luis Vives y su pacifismo (así Riba García en la Universidad de Zaragoza en 1933) o el Brocense y su enorme aportación lingüística, como expone en la Universidad de Salamanca el republicano catedrático Urbano González de la Calle en 1912. Otros motivos argumentales utilizados en este tipo de discursos inciden en la posición de la Universidad en relación a la intervención francesa en I808 (así, en Valencia, lo estudia Riba García en I9iI), o en los precedentes de una Universidad recién creada (1915), como sucede en Murcia, que debe remontarse a lejanos compromisos motivados por la apuesta que el rey Alfonso X el Sabio ya

42 «La situación es lamentable, y no cabe ignorar que ha sido deplorada, desde hace muchos años, en libros, en artículos y en discursos, por los mismos catedráticos. La enmienda no puede venir sino de la propia Universidad, cuando ésta disfrute de la necesaria independencia para decretarla; solo entonces será la universidad responsable de sus actos; solo entonces podrá justamente ser objeto de aplauso o de vituperio. Por ahora, la Universidad es menor de edad; está sometida a tutela; que el tutor responda» (cfr. Anexo, Bonilla y San Martin, Madrid, I9I4, p. I20). 
hizo en el siglo xiII por algunos establecimientos docentes creados entonces en aquella región (según explica el catedrático de Murcia y rector en ese momento Loustau en I92I).

Parece evidente que este epígrafe nos remite al uso legitimador de la historia, aquí de las propias universidades, para añorar tiempos pasados (que para algunos fueron mejores), para justificar la creación de nuevas universidades, para valorar aportaciones de grandes pensadores y profesores que pueden mantener vivas sus propuestas e intuiciones, o su brillante y respetable ejemplo académico y de vida entregada al estudio.

\section{Recapitulando}

La percepción que obtenemos de la Universidad española del primer tercio del siglo xx mediante los análisis procedentes de opiniones muy cualificadas, las de catedráticos, de élites de claustros universitarios, desde su experiencia científica y de vida, nos llevan a un espacio de confrontación de modelos de universidad no fáciles de integrar.

Existen en los claustros de las universidades españolas posiciones muy resistentes a las reformas que otros postulan como deseo, pero critican aceradamente la universidad del momento, como una de las causas del famoso desastre del 98. Pero al lado emergen de forma imparable, y con carácter mayoritario, otras posiciones partidarias de una universidad menos burocrática, más moderna, entusiasmada con la ciencia y la creación de conocimiento y saberes, que fija en pautas procedentes de países europeos (Alemania en particular) los criterios que ha de seguir esa deseada universidad. Se desea una universidad más viva y democrática, más abierta y sensible a los problemas de la calle, de la sociedad y de la industria, de la escuela, la cultura y de la enfermedad que precisan resolver millones de ciudadanos.

El estudio de un centenar largo de solemnes discursos inaugurales en las universidades españolas de esta etapa, de clara transición de modelos, nos ha permitido comprender el valor del trabajo histórico comparado que muchos oradores utilizan en el estrado y ponen por escrito en la edición de su discurso. Para argumentar novedades y deseos por cumplir en las mejoras universitarias, la contribución y argumentación histórica y comparada se erige en un rico patrimonio informativo y metodológico.

El uso del discurso del «día de la fiesta de la ciencia» se convierte para nosotros en un elemento informativo magnífico, escasamente utilizado en estudios de historia de la educación, que logra alcanzar valor en sí mismo cuando se utilizan muchos de ellos con carácter comparativo, pero que tiene también un valor complementario de otras fuentes de información para el estudio de la universidad y de la ciencia en la España del primer tercio del siglo xx. Los discursos son una fuente más, otro referente explicativo, junto a estudios monográficos de universidades, normativa universitaria, revistas científicas (el BILE, la Revista de Pedagogía, por ejemplo), pero reflejan un clima existente entre uno de los sectores más cualificados e influyentes en la Universidad española, como son los catedráticos de sus universidades. Han hablado las élites académicas sobre sus propias universidades. 
EL DISCURSO PEDAGÓGICO EN LA FIESTA DE LA CIENCIA

EN LA UNIVERSIDAD DESEADA: ESPAÑA I9OO-I936 JOSÉ MARÍA HERNÁNDEZ DÍAZ

No se puede afirmar de manera rotunda que se aprecie una evolución ascendente en la crítica y el deseo de renovación de la universidad en la España de inicios del siglo xx, tal como lo transmiten estos discursos. Pero sí observamos que de forma progresiva se abandona la retórica del lamento y la postración, de tono regeneracionista triste y "patriótico», y se va sustituyendo por una temática y un lenguaje que se aproxima mucho a los que rigen en otras muchas universidades europeas. La influencia ejercida por la JAE en este proceso nos parece innegable, porque permitió a algunos centenares de cualificados profesores españoles del primer tercio del siglo xx conocer otros climas científicos y pedagógicos, como el alemán, por ejemplo. La universidad española sigue mirándose, continúa ejerciendo la crítica sobre sí misma, pero desea encontrar otros parajes donde poderse expandir y cultivar en libertad para ponerse al servicio de la sociedad a la que se debe.

\section{Anexo}

Álvarez de Linera y Grund, Antonio: La verdad y la actitud de las universidades ante el relativismo moderno. Discurso pronunciado en la Universidad de La Laguna en la apertura del curso académico de I92I a I922, La Laguna, I92I, p. 53.

ANDRÉs Irueste, José: La enseñanza integral y la de las ciencias. Discurso pronunciado en la apertura del curso IgIo a IgII en la Universidad Central, Madrid, Impr. Colonial, I9IO, p. 44 .

Aprárz Buesa, Ángel de: Disciplinas estéticas y sus métodos en la universidad. Discurso pronunciado en la Universidad de Barcelona en la inauguración del curso I93I a 1932. Barcelona, Tip. Núñez, 1931, p. 30.

Aranda Millán, Francisco: Problemas biológico sociales de la universidad. Discurso pronunciado en la Universidad de Zaragoza en la apertura del curso 1931 a 1932, Zaragoza, Tip. La Académica, 1931, p. 45.

Aranzadi Unamuno, Telesforo: Vulgo y ciencia y sus relaciones. Discurso inangural pronunciado en la Universidad de Barcelona en la apertura del curso Igos a Igo6, Barcelona, Tip. La Académica, 1905, p. 17.

Arias de Velasco y Lúgigo, Jesús: Formar hombres libres. Discurso leído en la Universidad de Oviedo en la apertura del curso IgII a I9I2, Oviedo, Est. Tip. La Canóniga, I9II, p. 60.

Arias de Velasco y Lúgigo, Jesús: La enseñanza en Asturias. Discurso pronunciado en la Universidad de Oviedo en la inanguración del curso 1920 a I92I, Oviedo, Impr. Sucesores de Brid, 1920, p. 32.

BANQuÉ y Faliu, José: Algo que podemos hacer en España a favor de la cultura clásica. Florecimiento de los estudios helénicos, principalmente en el siglo actual. Discurso pronunciado en la Universidad de Barcelona en la apertura del curso IgII a IgI2, Barcelona, Tip. La Académica, I9II, p. 58.

BarRas y de Aragón, Francisco de la: De la Historia natural y su enseñanza. Discurso leído en la Universidad de Oviedo en la solemne apertura del curso académico 1907 a 1908, Oviedo, Est. Tip. Calle de la Canóniga, 1907, pp. $50+22$.

Bartolomé y del Cerro, Abelardo: De mi universidad, por y para ella. Discurso pronunciado en la Universidad de Valladolid en la apertura del curso 1933 a 1934, Valladolid, Tip. Cuesta, 1933, p. I5. 
BARtual y Moret, Juan: De ciertos males que agobian a la universidad española y de algunos de sus posibles remedios. Discurso leído en la Universidad de Valencia en la inauguración del curso I9I6 a I9I7, Valencia, Tip. Moderna, I9I6, p. 5I.

Berenguer Ballester, Ángel: La educación moral en nuestras universidades. Discurso inaugural leido en la solemne apertura del curso académico de IgIo a 1920 ante el claustro de la Universidad de Barcelona, Barcelona, Impr. La Académica, 1919, p. 28.

Bonet Bonet, Baldomero: La organización de la enseñanza. Discurso inaugural en la Universidad de Madrid en la apertura del curso 1907 a 1908, Madrid, Imprenta Colonial, 1907, p. 32.

Bonilla SAN Martín, Adolfo: La vida corporativa de los estudiantes españoles en sus relaciones con la historia de las universidades. Discurso leído en la Universidad de Madrid en la inauguración del curso I9I4 a I9IS, Madrid, Impr. Colonial, I9I4, p. I44.

Borobio Díaz, Patricio: Acción social de la universidad. Discurso leído en la Universidad de Zaragoza en la apertura del curso I9Io a I9II, Zaragoza, Tip. Casañal, ı9ıo, p. 5I.

BuÉn, Odón de: La enseñanza de la geografía en España. Discurso pronunciado en la Universidad de Barcelona en la apertura del curso Igog a I9Io, Barcelona, Tip. La Academia de Serra y Hmnos., 1909, p. 45.

Bustos Miguel, José de: Vocación para la enseñanza. Discurso leido en la Universidad de Salamanca en la apertura del curso 1922 a I923, Salamanca, Impr. Núñez, I922, p. 45.

Cabeza de León, Salvador: La disciplina en la Universidad de Santiago en el siglo XVIXVIII. Discurso inangural del curso IgI7 a IgI8 en la Universidad de Santiago, Santiago, Impr. Paredes, I917, p. 26.

Calamita Álvarez, Gonzalo: Causas del retraso de la ciencia quimica en España. Discurso pronunciado en la Universidad de Zaragoza en la inauguración del curso IgII a I9I2, Zaragoza, Tip. Casañal, I9II, p. 40.

Candau Pizarro, Feliciano: Decadencia de la universidad y nuevas orientaciones de la cultura. Discurso de apertura del curso I9I2 a I9I3 en la Universidad de Sevilla, Sevilla, Tip. de la Guía Oficial, r9ı2, p. 73.

Candela y Pla, Manuel: Rebabilitación social de España. Discurso de inauguración del curso Igoo a I9or en la Universidad de Valencia, Valencia, Tip. Domenech, I900, p. 87.

Canella Secades, Fermín: La enseñanza en el distrito universitario de Oviedo. Discurso pronunciado en la apertura del curso I9I3 a I9I4 en la Universidad de Oviedo. Oviedo, Impr. La Comercial, I9I3, p. 37.

Cañadas Domenech, Ramón: Espíritu de las actuales universidades. Discurso leído en la Universidad de Sevilla en la apertura del curso I92I a I922, Cádiz, Impr. Revista Médica, I92I, p. 49.

Cañizo García, Agustín del: Misión educativa y responsabilidad moral del catedrático. Discurso pronunciado en la apertura del curso 1927 a 1928 en la Universidad de Salamanca, Salamanca, Impr. Núñez, 1927, p. 30.

Casamada Maurí, Ramón: Necesidad de intensificar nuestras enseñanzas prácticas. Discurso pronunciado en la Universidad de Barcelona en la inauguración del curso 1930 a 1931, Barcelona, Impr. Núñez, I930, p. 22.

CASARES GIL, José: Nada contribuirá más a regenerar nuestra patria que una buena educación intelectual. Discurso inaugural del curso Igoo a Igor en la Universidad de Barcelona, Barcelona, Imp. Hijos de Jepús, 1900, p. 34.

Casares Gil, José: Necesidad de una reforma en la enseñanza de la Química. Discurso leído en la Universidad Central de Madrid en la inauguración del curso 1922 a 1923 , Madrid, Impr. Colonial, I922, p. 34.

Castell Miralles, Francisco: Reformas que han de dar resultados en la enseñanza universitaria, y especialmente en la sección de Químicas de la Facultad de Ciencias. Discurso 
EL DISCURSO PEDAGÓGICO EN LA FIESTA DE LA CIENCIA

EN LA UNIVERSIDAD DESEADA: ESPAÑA I9OO-I936 JOSÉ MARÍA HERNÁNDEZ DÍAZ

leído en la Universidad de Valencia en la apertura del curso Igos a 1906, Valencia, Est. Tip. Domenech, I905, p. 45.

Castro y de Castro, José de: De la lógica. Discurso pronunciado en la Universidad de Sevilla en la apertura del curso 1902 a I903, Sevilla, Papelera Sevillana, I902, p. 34.

Cerrada Martín, Félix: Algunas consideraciones sobre el problema de la segunda enseñanza en España. Discurso pronunciado en la Universidad de Zaragoza en la inauguración del curso I9I8 a I9I9, Zaragoza, Tip. Casañal, I9I8, p. 37.

Coll Pujol, Ramón: Que en las universidades de España se enseña y se enseña bien, y siempre se enseñó, siendo los analfabetos precisamente los que hablan mal de las cosas de España. Discurso leído en la Universidad de Barcelona en la inauguración del curso I9I3 a I9I4, Barcelona, Universidad de Barcelona, I9I3, p. I34.

Comín Moya, Francisco Javier: La libertad de cátedra. Discurso leído en la Universidad de Zaragoza en la inauguración del curso I9I2 a I9I3, Zaragoza, Tip. Casavial, I9I2, p. 46.

Cuenca Araújo, Enrique: Renovación de la profesión farmacéutica. Discurso leido en la inauguración del curso I9I8 a I9Io en la Universidad de Santiago, Santiago, Tip. Paredes, I9I8, p. 99.

Deleito Piñuela, José: La enseñanza de la historia en la universidad española y su posible reforma. Discurso de apertura del curso académico de 1918 a I9I9 en la Universidad Literaria de Valencia, Valencia, Tip. Moderna, 1918, p. 165.

Deulofeu Poch, José: Consideraciones acerca del estado actual de nuestra enseñanza universitaria. Discurso pronunciado en la Universidad de Santiago en la apertura del curso I9I4 a I9I5, Santiago, El Eco de Santiago, I9I4, p. 5I.

Díez Canseco, Laureano: La función de la universidad en la vida social. Discurso leído en la Universidad de Valladolid en la solemne inauguración del curso académico de 1908 a I909, Valladolid, Tip. Cuesta, I908, p. 83.

Díez SÁnchez, Ricardo: Cómo la libertad de enseñanza constituida sobre perfecta educación adquirida en el hogar doméstico y la escuela, y sobre sólidas bases de instrucción cientifica, ha de ejercer directa y saludable influencia en el desarrollo intelectual de los pueblos, pudiendo asi servir de eficaz remedio para combatir en lo sucesivo la degeneración que sufre nuestra sociedad actual. Discurso de apertura del curso Igora Igoz en la Universidad de Salamanca, Salamanca, Imp. Núñez, I9oı, p. 39.

Díez Tortosa, Juan Luis: Vida universitaria. Discurso pronunciado en la Universidad de Granada en la apertura del curso I9I9 a I920, Granada, Tip. Guevara, I919, p. 40.

Domenech Estapá, José: Conceptopedagógico de la ciencia matemática. Discurso pronunciado en la Universidad de Barcelona en la apertura del curso I904 a 1905, Barcelona, La Académica, 1904, p. 91.

Eleizegui López, Antonio: La reorganización de los estudios de farmacia. Discurso leído en la apertura del curso 1906 a 1907 en la Universidad de Santiago, Santiago, Impr. Paredes, 1906 , p. 42.

EsPerabé De Arteaga, Enrique: Qué ha de ser actualmente la universidad española, y en especial la de Salamanca para llevar a efecto la misión que le está encomendada. Discurso pronunciado en la Universidad de Salamanca en la apertura del curso 1924 a I925, Salamanca, Impr. Núñez, 1924, p. 27.

Espurz CAmobarde, Demetrio: Estudio de la física en sus diversos grados y de los medios más adecuados para su respectiva enseñanza. Discurso pronunciado en la Universidad de Oviedo en la apertura del curso I909 a I9I0, Oviedo, Est. Tip. 1909, p. 87.

Fernández Baños, Olegario: Previsiones cientificas. Discurso inangural leído en la Universidad de Santiago para el curso 1929 a 1930, Santiago, Universidad de Santiago, 1929, p. 46.

Fernández DEL Riego, Francisco: La universidad gallega ante el momento actual. Discurso leído en la Universidad de Santiago en la apertura del curso 1933 a 1934, Santiago, Tip. Paredes, I933, p. I4. 
FERnÁNDEz Rodríguez, Obdulio: Relaciones entre la universidad y la industria. Discurso leído en la Universidad Central de Madrid en la inauguración del curso IgI7 a I9I8. Madrid, Impr. Colonial, I917, p. 72.

Fresno y Pérez del Villar, Carlos del: Enseñanza de la química en las universidades españolas. Discurso pronunciado en la Universidad de Oviedo en la apertura del curso I930 a I93I, Oviedo, Universidad de Oviedo, 1930, p. 33.

Frontera Aurrecoechea, José María: Algunas consideraciones sobre la urgente necesidad de implantar la enseñanza profesional obligatoria en España como base del inmediato mejoramiento de la industria y el comercio. Discurso pronunciado en la Universidad de Oviedo en la inauguración del curso I9Is a I9I6, Oviedo, Suc. de Adolfo Brid, I9I5, p. 59.

Galcerán Cifuentes, Isaac: Del estudio en las universidades de la Economía Política. Discurso pronunciado en la Universidad de Oviedo en la apertura del curso académico I9I8 a I9I9, Oviedo, Impr. Brid, I9I8, p. 55.

García Alas y G. Argüelles, Leopoldo: Situación y reorganización de la enseñanza superior. Discurso pronunciado en la Universidad de Oviedo en la inauguración del curso 1922 a 1923, Oviedo, Impr. Flórez, I922, p. 54.

García García, José: El momento universitario. Discurso pronunciado en la Universidad de Madrid en la apertura de curso I93I a 1932, Madrid, Impr. Colonial, I931, p. I8.

García VArela, Antonio: Aspectos de la enseñanza en la Facultad de Ciencias, como parte integrante de la universidad. Discurso pronunciado en la Universidad de Santiago en la inauguración del curso I9II a I9I2, Santiago, Impr. Paredes, I9II, p. 52.

GARCía VÉLEZ, José: Algunos aspectos que presenta el problema de la valoración biológica de los medicamentos complejos. Discurso pronunciado en la Universidad de Granada en la inauguración del curso 1935 a 1936, Granada, Impr. Paulino Traveset, 1935, p. 42.

GARrido Osorio, Manuel: El estado de la filosofía griega antes de Sócrates y los medios empleados por éste para restaurarla, indicando a la vez algunas de las ventajas que de ellos puede reportar la enseñanza. Discurso pronunciado en la Universidad de Granada en la apertura del curso I900 a I90I, Granada, Imp. Indalecio Ventura, I900, p. 55.

Gaspar Arnal, Teófilo: Algunas estampas del acuerdo entre la economía y la investigación cientifica. Discurso pronunciado en la Universidad de La Laguna en la inauguración del curso I933 a 1934, Madrid, Tip. de Victoriano Suárez, I933, p. I09.

Gil Fagoaga, Lucio: La selección profesional de los estudiantes. Discurso leído en la Universidad Central de Madrid en la inauguración del curso I929 a 1930, Madrid, Universidad de Madrid, 1929, p. I86.

Gil MorTE, Adolfo: El presente de las universidades y el porvenir en sus relaciones con el régimen autonómico. Discurso leido en la Universidad de Valencia en la inauguración del curso I920 a I92I, Valencia, Tip. Ferrandis, I920, p. 51.

Giral Pereira, José: Posición de la universidad ante el problema industrial. Discurso leído en la apertura del curso I9I8 a I9Ig en la Universidad de Salamanca, Salamanca, Impr. El Salmantino, i918, p. 63.

Goizueta Díaz, Jesús: El segundo factor de la enseñanza. Discurso leído en la Universidad de Barcelona en la inauguración del curso I9Is a I9I6, Barcelona, Tip. La Académica, I9I5, p. 37 .

Gómez IzQuierdo, Alberto: La inauguración solemne del curso como fiesta de la labor intelectual. Discurso pronunciado en la Universidad de Granada en la inauguración del curso I920 a I92I, Granada, Tip. López Guevara, 1920, p. 47.

GonzÁlez de la CAlle, Urbano: Estudio de la vida académica y profesional del Brocense en nuestra Escuela. Discurso pronunciado en la Universidad de Salamanca en la inauguración del curso I9I2 a I9I3, Salamanca, Tip. Núñez, I9I2, p. I92. 
EL DISCURSO PEDAGÓGICO EN LA FIESTA DE LA CIENCIA

EN LA UNIVERSIDAD DESEADA: ESPAÑA I9OO-I936

JOSÉ MARÍA HERNÁNDEZ DÍAZ

GonzÁlez Meneses y Jiménez, José: Elementos que integran la luch a contra la mortalidad de la primera infancia en España. Discurso de apertura del curso académico de 1935 a I936 en la Universidad de Sevilla, Sevilla, J. Mejías, I935, p. 52.

Gonzalvo París, Luis: Carácter, elaboración y enseñanza de los estudios históricos. Discurso leído en la Universidad Literaria de Valencia en la inauguración del curso 1914 a I9I5, Valencia, Tip. Moderna, I9I4, p. 36.

HaZAÑAS Y LA RÚA, Joaquín: La vida escolar universitaria en Sevilla (XVI-XVIII). Discurso pronunciado en la Universidad de Sevilla en la inauguración del curso 1907 a 1908, Sevilla, Papelería Sevillana, I907, p. 20.

Hazañas y la Rúa, Joaquín: La Universidad de Sevilla. Discurso pronunciado en la Universidad de Sevilla en la inauguración del curso 1922 a 1923, Sevilla, Tip. Eulogio de las Heras, i922, p. 33.

Iranzo, Juan Enrique: La medicina y los médicos en la universidad de Zaragoza. Discurso pronunciado en la Universidad de Zaragoza en la inauguración del curso I9I4 a I9IS, Zaragoza, Imp. Heraldo, i9ı4, p. 38.

IzQuierdo Gómez, Juan Antonio: De la enseñanza de la física en la Facultad de Ciencias Quimicas. Discurso leído en la Universidad de Valencia en la inauguración del curso I9I7 a I9I8, Valencia, Tip. Moderna, I9I7, p. 64.

Jiménez de Cisneros Hervás, Diego: Lo hipotético en las ciencias experimentales. Discurso leído en la Universidad de La Laguna en la inauguración del curso 1924 a 1925, La Laguna, Impr. M. Curbelo, I924, p. 69.

Lasso de la Vega Cortezo, Javier: Feminismo. Discurso pronunciado en la Universidad de Sevilla en la inauguración del curso 1904 a 1905, Sevilla, Papelería Sevillana, 1904, p. 38.

LÁzaro Ibiza, Blas: Problemas de la educación y la cultura patrias. Discurso pronunciado en la Universidad Central de Madrid en la apertura del curso I902 a I903, Madrid, Impr. Colonial, I902, p. 98.

Ledo Eguiarte, Eduardo: La importancia de la educación infantil. Sus progresos en el XIX y su estado en España. Discurso pronunciado en la Universidad de Valladolid en la apertura del curso 1903 a 1904, Valladolid, Impr. Cuesta, 1903, p. 57.

Lово Gómez, Ruperto: La universidad en sus relaciones sociales. Discurso pronunciado en la Universidad de Santiago en la apertura del curso IgIg a I920, Santiago, Impr. Paredes, I9I9, p. 43.

López Capdepón, José: Cómo era, es y debía ser la enseñanza práctica de la química en la Facultad de Farmacia. Discurso pronunciado en la Universidad de Barcelona en la apertura del curso I920 a I92I, Barcelona, La Académica, I920, p. 55.

López Domínguez, Manuel: En la paz como en la guerra, la vitalidad de una nación es función directa de la de su institución universitaria. Discurso pronunciado en la Universidad de Sevilla en la apertura del curso I920 a I92I, Sevilla, Imp. Eulogio de las Eras, I920, p. 52.

López PRIeTO, Ramón: Selección de los alumnos de Medicina. Valor que en esta selección y en la formación profesional puede concederse a la Anatomía. Discurso leído en la Universidad de Valladolid en la inauguración del curso I93I a 1932, Valladolid, Tip. Cuesta, I93I, p. 9I.

López R. Gómez, Nicolás: Necesidad de la instrucción social, politica y jurídica para el recto cumplimiento de los deberes y ejercicio de los derechos. Discurso pronunciado en la Universidad de Valladolid en la apertura del curso I906 a 1907, Valladolid, Impr. Cuesta, 1906, p. 59.

Loustau Gómez de Membrillera, José: La Universidad de Murcia y el organismo social universitario. Discurso leído en la inauguración del curso de I92I a 1922 en la Universidad Literaria de Murcia, Murcia, José A. Giménez, I921, p. 86. 
Lozano Rey, Luis: Reconstitución de la raza sobre la base de la ciencia. Discurso pronunciado en la Universidad de Madrid en la apertura del curso I9IS a I9I6, Madrid, Imp. Colonial, I9I5, p. 54.

Macher VILAR, Pascual: Si cual demuestra la historia las universidades son las fuentes de todo progreso y las escuelas de espíritu público, urge que nuestros gobiernos se preocupen del estado en que se encuentran las españolas. Discurso pronunciado en la Universidad de Granada en la apertura del curso 1903 a 1904, Granada, Tip. Indalecio Ventura, I903, p. 70.

Madinaveitia Tabuyo, Antonio: La enseñanza de la química orgánica. Discurso leido en la solemne inauguración del curso académico de 1927 a 1928 en la Universidad de Madrid, Madrid, Universidad Central, 1927, p. 47.

Maldonado de Guevara, Francisco: Salamanca y su universidad. Discurso pronunciado en la Universidad de Salamanca en la apertura del curso 1933 a 1934, Salamanca, Impr. Núñez, I933, p. 27.

Maldonado de Guevara y Fernandez Ocampo, Luis de: Elogio de Dorado Montero y otros catedráticos de Salamanca. Discurso pronunciado en la Universidad de Salamanca en la apertura del curso I919 a I920, Salamanca, Impr. Núñez, 1919, p. 38.

Martín González, Pedro Nicanor: Universidad. Discurso de apertura del curso 1932 a 1933 en la Universidad de Madrid, Madrid, Impr. Colonial, I932, p. I8.

MARTOS DE LA FuenTE, José: La universidad española hasta el siglo XVIII y sus rasgos característicos. Discurso leido en la Universidad de Granada en la apertura del curso 1928 a 1929, Granada, Tip. López de Guevara, 1928, p. 44.

Melón y Ruiz de Gordejuela, Amando: Alejandro von Humboldt en América española. Discurso leido en la Universidad de Valladolid en la inauguración del curso 1932 a 1933, Valladolid, Tip. Cuesta, I932, p. IOI.

Meneu Meneu, Pascual: Estudios semíticos y su reorganización en España. Discurso pronunciado en la Universidad de Salamanca en la apertura del curso I9I6 a 19I7, Salamanca, Impr. Núñez, i916, p. 40.

Miral López, Domingo: La crisis de la universidad. Oración inaugural del curso 1908 a I909 en la Universidad de Salamanca, Salamanca, Impr. El Castellano, 1908, p. 92.

Miral López, Domingo: Bases para una pedagogía aragonesa. Discurso leído en la Universidad de Zaragoza en la apertura del curso I9I7 a I9I8, Zaragoza, Tip. Casañal, I9I7, p. Io8.

Moliner Nicolás, Francisco: La dimisión de la cátedra. Discurso inaugural del curso 1904 a Igos en la Universidad de Valencia, Valencia, Impr. Domenech, 1904, p. 78.

Mollá Rodrigo, Rafael: La enseñanza de la medicina, aspecto de la educación y cultura nacional. Discurso leído en la apertura del curso 1928 a 1929 en la Universidad de Madrid, Madrid, Impr. Colonial, I928, p. 82.

Mur Ainsa, José: Pedagogía matemática. Discurso leido en la solemne apertura del curso académico de IgIo a IوII en la Universidad Literaria de Oviedo, Oviedo, Impr. Brid, I9IO, p. I37.

Murueta Goyena, Federico: Rápido examen de las modernas manifestaciones de la escuela americana seguido de un brevísimo paralelo entre ella y la escuela europea. Discurso leido en la Universidad de Valladolid en la apertura del curso I9IS a I9I6, Valladolid, Impr. Cuesta, I9I5, p. I30.

Nacle Herrera, Juan: Consideraciones acerca de los métodos y plan de enseñanza de la Facultad de Farmacia. Discurso pronunciado en la Universidad de Granada en la apertura del curso 1930 a 1931, Granada, Tip. López Guevara, 1930, p. 30.

Novoa López, Jesús: Métodos de enseñanza en Medicina. Discurso leído en la Universidad de Santiago en la inauguración del curso I904 a 1905, Santiago, Impr. Paredes, 1904, p. 73 . 
EL DISCURSO PEDAGÓGICO EN LA FIESTA DE LA CIENCIA

EN LA UNIVERSIDAD DESEADA: ESPAÑA I9OO-I936

JOSÉ MARÍA HERNÁNDEZ DÍAZ

Olóriz Ortega, Federico: Material clínico de enseñanza de que disponen las Facultades de Medicina en España. Discurso leído en la Universidad de Granada en la apertura del curso 1927 a 1928, Granada, Tip. López Guevara, 1927, p. 54.

Onís SÁnchez, Federico de: Acerca de la universidad. Discurso pronunciado en la apertura del curso 1912 a I9I3 en la Universidad de Oviedo, Oviedo, Imprenta de Adolfo Brid, I9I2, p. 6I.

Onís Sánchez, Federico de: El español en Estados Unidos. Discurso leído en la Universidad de Salamanca en la inauguración del curso académico I920 a I92I, Salamanca, Impr. Núñez, I920, p. 34.

Otтo Escudero, Nicolás de: Valor jurídico y social del nombre en sus diversas manifestaciones. Discurso leido en la solemne apertura del curso académico de 1928 a 1929 en la Universidad de Murcia, Barbastro, Castillón, I928, p. 88.

PAREJA GARRIDO, José: Estado actual de la enseñanza de la medicina en nuestro país y de la necesidad y dirección de su reforma. Discurso pronunciado en la Universidad de Granada en la inauguración del curso I9II a I9I2, Granada, Impr. López Guevara, I9II, p. 3I.

PAREJA GARRIDO, José: Algunas reflexiones sobre el estado actual de nuestras universidades. Discurso pronunciado en la Universidad de Granada en la apertura del curso 1922 a 1923, Granada, Tip. López Guevara, 1922, p. 34.

Párraga Acuña, Celestino: A las relaciones existentes entre los objetos de las distintas ciencias, debe corresponder la misma relación entre los cultivadores de ellas. Discurso leído en la inauguración del curso 1907 a Igo8 en la Universidad de Sevilla, Cádiz, Tip. Revista Médica, 1907, p. 67.

Pastor Pellicer, Manuel: Algunas reflexiones sobre las reformas que reclaman la enseñanza y el ejercicio de la terapéutica. Discurso pronunciado en la Universidad de Zaragoza en la inauguración del curso 1902 a 1903, Zaragoza, Tip. Viuda de Ariño, 1902, p. 74.

Pérez Bueno, Fernando: Llagas de la enseñanza. Discurso pronunciado en la Universidad de Oviedo en la inauguración del curso 1905 a 1906, Oviedo, Tip. Brid, I905, p. 59.

Perez Carballo, Francisco: Estampa de universidad. Discurso leido en la universidad de Madrid en la apertura del curso I933 a I934, Madrid, Impr. Colonial, I933, p. 54.

Pérez Martín, Arturo: Ensayo de política pedagógica urgente. Discurso pronunciado en la Universidad de Oviedo en la apertura del curso 1904 a 1905, Oviedo, Impr. Brid, I904, p. 88.

Peset y Cervera, Vicente: ;Amemus patriam! La influencia española en la cultura mundial. Discurso pronunciado en la Universidad Literaria de Valencia en la inauguración del curso I924 a I925, Valencia, Impr. Hijo de Vives Mora, 1924, p. 67.

Piñerúa Álvarez, Eugenio: Educación moral o ética. Discurso pronunciado en la Universidad de Madrid en la inauguración del curso I920 a I92I, Madrid, Impr. Colonial, I920, p. 65.

Población Sánchez, Casimiro: Algunas orientaciones para la reforma de la enseñanza médica. Discurso leido en la solemne apertura del curso académico de 1931 a 1932 en la Universidad de Salamanca, Salamanca, Impr. Núñez, I93I, p. 30.

Puente Sánchez, Carlos: La enseñanza en general y particularmente la de química. Discurso inangural del curso 1922 a 1923 en la Universidad de Santiago, Santiago, Tip. Paredes, I922, p. 43.

Requejo Alonso, Prudencio: Pedagogía del Derecho Mercantil. Discurso pronunciado en la inauguración del curso IgII-IgIz en la Universidad de Salamanca, Salamanca, Impr. Popular, I9II, p. 59.

Riba García, Carlos: La Universidad valentina en los años de la Guerra de la Independencia (I807-I8I5). Datos y documentos para su historia. Discurso leído en la solemne apertura de los estudios del año académico de IgIo a IgII de la Universidad de Valencia, Valencia, I9II, p. I47. 
Riba García, Carlos: Luis Vives y el pacifismo. Discurso de inauguración del curso 1933 a I934 en la Universidad de Zaragoza, Zaragoza, Ed. Universidad, I933, p. 79.

Ríos UrRuti, Fernando de los: La crisis de la democracia. Discurso inaugural del curso I9I7 a I9I8 pronunciado en la Universidad de Granada, Granada, Tip. Guevara, I917, p. 43.

Rivas Mateos, Marcelo: El profesorado universitario. Discurso de apertura en la Universidad Central de Madrid para el curso I9I2 a I9I3, Madrid, Impr. Colonial, I9I2, p. 70.

Rodrigo Lavín, Leonardo: Avances necesarios en la enseñanza médica. Discurso leido en la Universidad de Sevilla en la apertura del curso 1926 a 1927, Cádiz, Tip. Ordóñez, I926, p. 85 .

Rodríguez Ávila, Manuel: Causa del atraso de los españoles en la química. Discurso leído en la Universidad de Granada en la inanguración del curso Igog a I9Io, Granada, Impr. de Indalecio Ventura López, 1909, p. 34.

Rodríguez Fernández, Ildefonso: El racionalismo y las ciencias. Discurso leído en la Universidad Central de Madrid en la inauguración del curso I9I3 a I9I4, Madrid, Impr. Colonial, I9I3, p. 79.

Rodríguez Pinilla, Hipólito: La psicología del estudiante o estado psicológico del niño y del adolescente. Discurso leído en la inauguración del curso académico de 1913 a I9I4 en la Universidad de Salamanca, Salamanca, Tip. Popular, ı193, p. 26.

Rodríguez Risueño, Emiliano: Modernos estudios de bilogía y enseñanza general y experimental. Discurso pronunciado en la Universidad de Valladolid en la inanguración del curso I901 a 1902, Valladolid, Imp. Cuesta, I901, p. 63.

Román Retuerto, Emilio: Aspiraciones de la universidad española y especialmente la de Salamanca. Discurso pronunciado en la Universidad de Salamanca en la apertura del curso 1926 a 1927, Salamanca, Impr. Núñez, 1926, p. 32.

Roquero Martínez, José: La enseñanza en general y en particular de la Medicina. Discurso pronunciado en la Universidad de Sevilla en la inauguración del curso Igog a I9Io, Sevilla, Papelería Sevillana, 1909, p. 42.

Rovira Carreró, Isaac: Concepción Arenal. Su labor cientifica desde los puntos de vista penal y penitenciario: notas para un estudio crítico. Discurso inangural del curso 1926-27 en la Universidad de Santiago, Santiago, Imp. Paredes, 1926, p. 190.

Royo González, Miguel: Problemas universitarios. Discurso pronunciado en la Universidad de Sevilla en la inanguración del curso Igrg a I920, Sevilla, Impr. Eulogio de las Heras, I919, p. 55.

Sánchez Gallego, Mariano: El descrédito del Derecho. Discurso pronunciado en la Universidad de Murcia en la inauguración del curso 1919 a 1920, Madrid, Impr. La Enseñanza, 1919, p. 46.

SANTOS FERnÁNDEZ, Víctor: Régimen escolar y medios de evitar y corregir las enfermedades escolares. Discurso leído en la Universidad de Valladolid en la inauguración del curso I909 a I9I0, Valladolid, Tip. Cuesta, I909, p. 88.

Segovia Corrales, Isidro de: De la enseñanza en España. Sus defectos y sus remedios. Discurso leido en la inauguración del curso Igog a Igro en la Universidad de Salamanca, Salamanca, Impr. Núñez, I909, p. 64.

Serra Rafols, Elías: Una universidad medieval, el Estudio General de Lérida. Discurso pronunciado en la Universidad de La Laguna en la apertura del curso I93I a 1932, Madrid, Victoriano Suárez, I93I, p. 94.

Sesé Villanueva, Mariano: Alocución de animación al estudio. Oración inaugural del curso rgıо a I9II en la Universidad de Salamanca, Salamanca, Tip. Calatrava, I9Io, p. 2I.

Simonena Zabalegui, Antonio: De la intensión universitaria. Discurso pronunciado en la Universidad de Valladolid en la inauguración del curso Igos a 1906, Valladolid, Tip. Cuesta, 1905, p. 82. 
EL DISCURSO PEDAGÓGICO EN LA FIESTA DE LA CIENCIA

EN LA UNIVERSIDAD DESEADA: ESPAÑA I9OO-I936

JOSÉ MARÍA HERNÁNDEZ DÍAZ

Simonena Zabalegui, Antonio: Del orden en la universidad. Discurso pronunciado en la Universidad Central de Madrid en la apertura del curso 1923 a I924, Madrid, Impr. Colonial, I923, p. 7I

Soriano Sánchez, Manuel: Métodos de enseñanza, programas, libros de texto y disciplina escolar. Discurso pronunciado en la Universidad de Barcelona en la apertura del curso 1906 a 1907, Barcelona, La Académica, 1906, p. 23.

Téllez de Meneses SÁnchez, José: La Universidad Salmantina y su riqueza bibliográfica. Discurso pronunciado en la Universidad de Salamanca en la apertura del curso académico de I925 a 1926, Salamanca, Imp. Núñez, 1925, p. 3I.

Testor Pascual, Pascual: Política universitaria. Discurso leído en la solemne inanguración del curso IgII a IgI2 de la Universidad Literaria de Valencia, Valencia, Tip. Moderna, I9II, p. 77.

Tormo Monzó, Elías: Las Bellas Artes, nueva entre las disciplinas universitarias. Discurso de inauguración del curso Igog a Igro en la Universidad de Madrid, Madrid, Impr. Colonial, I909, p. 43.

Torre del Cerro, Antonio de la: Provisión de cátedras en la Universidad de Barcelona de I559 a I596. Discurso pronunciado en la Universidad de Barcelona en la inanguración del curso I926 a 1927, Barcelona, Núñez y Cos, 1926, p. 26.

Torremocha Téllez, Lorenzo: La enseñanza experimental de la medicina en España. Discurso pronunciado en la apertura del curso 1927 a 1928 de la Universidad de Valladolid, Valladolid, Impr. Cuesta, 1927, p. 62.

Torres Campos, Manuel: De los estudiantes, de sus asociaciones y de los medios adoptados con el objeto de protegerlos. Discurso pronunciado en la Universidad de Granada en la apertura del curso 1907 a 1908, Granada, Indalecio Ventura López, 1907, p. 23.

Traviesas, Manuel Miguel: Finalidad que corresponde a una Facultad de Derecho. Medios. Los estudios de latín y griego como instrumentos de educación y de trabajo jurídico cientifico. Valor del derecho romano para este efecto. Discurso pronunciado en la apertura del curso académico de IgI4 a IgIs en la Universidad de Oviedo, Oviedo, Est. Tip. Calle Canónigo, I9ı4, p. 69.

Unamuno, Miguel de: Exhortación a la juventud estudiante. Discurso pronunciado en la Universidad de Salamanca en la apertura del curso Igoo a Igor, Salamanca, Imp. Núñez, I900, p. I6.

Unamuno, Miguel de: La palabra. Discurso pronunciado en la Universidad de Salamanca en la apertura del curso I934 a 1935, Salamanca, Imp. Núñez, 1934, p. I7.

UREÑA Y SMENJAUd, Rafael de: Observaciones acerca del desenvolvimiento de los estudios de Historia del Derecho español. Discurso pronunciado en la Universidad Central de Madrid en la apertura del curso I906 a I907, Madrid, Impr. Colonial, I906, p. I56.

Valentí Vivo, Ignacio: La función social de la universidad moderna. Discurso pronunciado en la Universidad de Barcelona en la inauguración del curso 1903 a 1904, Barcelona, Imp. J. Jepús, I903, p. 31.

Vargas Martínez, Andrés: Educación. Discurso pronunciado en la Universidad de Barcelona en la inauguración del curso I9I8 a I9I9, Barcelona, Tip. La Academia de Serra, I9I8, p. 93.

Vijande y Fernández Luanco, José María: La Facultad de Ciencias de Oviedo durante su primera época. Discurso leído en la Universidad de Oviedo en la apertura de curso I9I7 a I9I8, Oviedo, Imp. Brid, I917, p. 78.

Vilariño Magdalena, Eduardo: La enseñanza del Derecho en las universidades. Discurso leido en la universidad de Santiago en la solemne inauguración del curso académico de Igog a I9Io, Santiago, Impr. Paredes, 1909, p. 74. 
Zabala Lera, Pío: La autonomía universitaria. Discurso leído en la Universidad Central de Madrid en la apertura del curso I9I9 a I920, Madrid, Imp. Universidad, I919, p. 67.

Zumalacárregui y Prat, José María: Misión de la Universidad en la vida económica contemporánea. Discurso leído en la solemne inauguración del curso IgIo a Igzo de la Universidad Literaria de Valencia, Valencia, Tip. Moderna, I919, p. Iоo. 